\title{
The representation theorem of persistence revisited and generalized
}

\author{
René Corbet $^{1}\left[\right.$ [ $\cdot$ Michael Kerber ${ }^{1}(1)$
}

Received: 9 November 2017 / Accepted: 15 June 2018 / Published online: 9 July 2018

(c) The Author(s) 2018

\begin{abstract}
The Representation Theorem by Zomorodian and Carlsson has been the starting point of the study of persistent homology under the lens of representation theory. In this work, we give a more accurate statement of the original theorem and provide a complete and self-contained proof. Furthermore, we generalize the statement from the case of linear sequences of $R$-modules to $R$-modules indexed over more general monoids. This generalization subsumes the Representation Theorem of multidimensional persistence as a special case.
\end{abstract}

Keywords Computational topology · Persistence modules · Persistent homology · Representation theory

Mathematics Subject Classification 06F25 1 16D90 $\cdot 16 \mathrm{~W} 50 \cdot 55 \mathrm{U} 99 \cdot 68 \mathrm{~W} 30$

\section{Introduction}

Persistent homology, introduced by Edelsbrunner et al. (2002), is a multi-scale extension of classical homology theory. The idea is to track how homological features appear and disappear in a shape when the scale parameter is increasing. This data can be summarized by a barcode where each bar corresponds to a homology class that appears in the process and represents the range of scales where the class is present. The usefulness of this paradigm in the context of real-world data sets has led to the term topological data analysis; see the surveys (Carlsson 2009; Ghrist 2008; Edelsbrunner and Morozov 2012; Vejdemo-Johansson 2014; Kerber 2016) and textbooks (Edelsbrunner and Harer 2010; Oudot 2015) for various use cases.

René Corbet

corbet@tugraz.at

Michael Kerber

kerber@tugraz.at

1 Institute of Geometry, Graz University of Technology, Kopernikusgasse 24, 8010 Graz, Austria 
A strong point of persistent homology is that it can be defined and motivated both in geometric and in algebraic terms. For the latter, the main object are persistence modules. In the simplest case, such a persistence module consists of a sequence of $R$-modules indexed over $\mathbb{N}$ and module homomorphisms connecting consecutive modules, as in the following diagram:

$$
M_{0} \stackrel{\varphi_{0}}{\longrightarrow} M_{1} \stackrel{\varphi_{1}}{\longrightarrow} \ldots \stackrel{\varphi_{i-1}}{\longrightarrow} M_{i} \stackrel{\varphi_{i}}{\longrightarrow} M_{i+1} \stackrel{\varphi_{i+1}}{\longrightarrow} \ldots
$$

A persistence module as above is of finitely generated type if each $M_{i}$ is finitely generated and there is an $m \in \mathbb{N}$ such that $\varphi_{i}$ is an isomorphism for all $i \geq m$. Under this condition, Zomorodian and Carlsson (2005) observed that a persistence module can be expressed as single module over the polynomial ring $R[t]$ :

ZC-Representation Theorem (Theorem 3.1 in Zomorodian and Carlsson 2005) Let $R$ be a commutative ring with unity. The category of persistence modules of finitely generated type ${ }^{1}$ over $R$ is equivalent to the category of finitely generated graded modules over $R[t]$.

The importance of this equivalence stems from the case most important for applications, namely if $R$ is a field. In this case, graded $R[t]$-modules, and hence also persistence modules of finitely generated type, permit a decomposition

$$
\left.\left(\bigoplus_{i=1}^{n} \Sigma^{\alpha_{i}} R[t]\right) \oplus\left(\bigoplus_{j=1}^{m} \Sigma^{\beta_{j}} R[t] /\left(t^{n_{j}}\right)\right)\right)
$$

where $\Sigma \cdot$ denotes a shift in the grading. The integers $\alpha_{i}, \beta_{j}, n_{j}$ give rise to the aforementioned barcode of the persistence module; see Zomorodian and Carlsson (2005) for details. Subsequent work studied the property of more general persistence modules, for instance, for modules indexed over any subset of $\mathbb{R}$ (and not necessarily of finite type) (Chazal et al. 2016; Crawley-Boevey 2015) and for the case that the $M_{i}$ and $\varphi_{i}$ are replaced with any objects and morphisms in a target category (Bubenik et al. 2015; Bubenik and Scott 2014).

Given the importance of the ZC-Representation Theorem, it is remarkable that a comprehensive proof seems not to be present in the literature. In Zomorodian and Carlsson (2005), the authors assign an $R[t]$-module to a persistence module of finite type and simply state:

The proof is the Artin-Rees theory in commutative algebra (Eisenbud 1995).

In Zomorodian's textbook (Zomorodian 2009), the same statement is accompanied with this proof (where $\alpha$ is the assignment mentioned above):

\footnotetext{
${ }^{1}$ In Zomorodian and Carlsson (2005), the term "finite type" is used instead, but we renamed it here as we will define another finiteness condition later.
} 
It is clear that $\alpha$ is functorial. We only need to construct a functor $\beta$ that carries finitely generated non-negatively graded $k[t]$-modules [sic] to persistence modules of finite[ly generated] type. But this is readily done by sending the graded module $M=\bigoplus_{i=0}^{\infty} M_{i}$ to the persistence module $\left\{M_{i}, \varphi_{i}\right\}_{i \in \mathbb{N}}$ where $\varphi_{i}: M^{i} \rightarrow M^{i+1}$ is multiplication by t. It is clear that $\alpha \beta$ and $\beta \alpha$ are canonically isomorphic to the corresponding identity functors on both sides. This proof is the Artin-Rees theory in commutative algebra (Eisenbud 1995).

While that proof strategy works for the most important case of fields, it fails for "sufficiently" bad choices of $R$, as the following example shows:

Let $R=\mathbb{Z}\left[x_{1}, x_{2}, \ldots\right]$ and consider the graded $R[t]$ module $M:=\bigoplus_{i \in \mathbb{N}} M_{i}$ with $M_{i}=R /<x_{1}, \ldots, x_{i}>$ where multiplication by $t$ corresponds to the map $M_{i} \rightarrow M_{i+1}$ that assigns $p \bmod x_{i}$ to a polynomial $p$. $M$ is generated by $\{1\}$. However, the persistence module $\beta(M)$ as in Zomorodian's proof is not of finitely generated type, because no inclusion $M_{i} \rightarrow M_{i+1}$ is an isomorphism.

This counterexample raises the question: what are the requirements on the ring $R$ to make the claimed correspondence valid? In the light of the cited Artin-Rees theory, it appears natural to require $R$ to be a Noetherian ring (that is, every ascending chain of ideals becomes stationary), because the theory is formulated for such rings only; see Eisenbud (1995) and Greuel and Pfister (2007). Indeed, as carefully exposed in the master's thesis of the first author (Corbet 2016), the above proof strategy works under the additional assumption of $R$ being Noetherian. We sketch the proof in Appendix 3.

Our contributions As our first result, we prove a generalized version of the $\mathrm{ZC}$ Representation Theorem. In short, we show that the original statement becomes valid without additional assumptions on $R$ if "finitely generated type" is replaced with "finitely presented type" (that is, in particular, every $M_{i}$ must be finitely presented). Furthermore, we remove the requirement of $R$ being commutative and arrive at the following result.

Theorem Let $R$ be a ring with unity. The category of persistence modules of finitely presented type over $R$ is isomorphic to the category of finitely presented graded modules over $R[t]$.

The example from above does not violate the statement of this theorem because the module $M$ is not finitely presented. Also, the statement implies the ZC-Representation Theorem for commutative Noetherian rings, because if $R$ is commutative with unity and Noetherian, finitely generated modules are finitely presented.

Our proof follows the same path as sketched by Zomorodian, using the functors $\alpha$ and $\beta$ to define a (straight-forward) correspondence between persistence modules and graded $R[t]$-modules. The technical difficulty lies in showing that these functors are well-defined if restricted to subclasses of finitely presented type. It is worth to remark that our proof is elementary and self-contained and does not require Artin-Rees theory at any point. We think that the ZC-Representation Theorem is of such outstanding importance in the theory of persistent homology that it deserves a complete proof in the literature. 


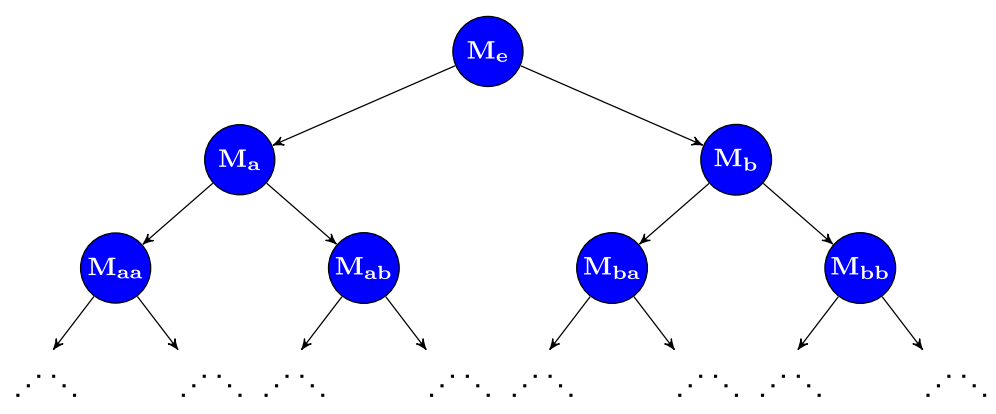

Fig. 1 Graphical illustration of a generalized persistence module. The underlying monoid is the set of words over $\{a, b\}$. For each monoid element, the persistence module contains an $R$-module, and for each arrow, the module contains a homomorphism (which is not specified in the figure)

As our second result, we give a Representation Theorem for a more general class of persistence modules. We work over an arbitrary ring $R$ with unity and generalize the indexing set of persistence modules to a monoid $(G, \star) .{ }^{2}$ We consider a subclass which we call "good" monoids in this work (see Sect. 4 for the definition and a discussion of related concepts). Among them is the case $\mathbb{N}^{k}$ corresponding to multidimensional persistence modules, but also other monoids such as $\left(\mathbb{Q}_{\geq 0},+\right)$, $(\mathbb{Q} \cap(0,1], \cdot)$ and the non-commutative word monoid as illustrated in Fig. 1. It is not difficult to show that such generalized persistence modules can be isomorphically described as a single module over the monoid ring $R[G]$.

Our second main result is that finitely presented graded modules over $R[G]$ correspond again to generalized persistence modules with a finiteness condition. Specifically, finiteness means that there exists a finite set $S$ of indices (i.e., elements in the monoid) such that for each monoid element $g$ with associated $R$-module $R_{g}$, there exists an $s \in S$ such that each map $R_{s} \rightarrow R_{\tilde{g}}$ is an isomorphism, whenever $\tilde{g}$ lies between $s$ and $g$.

For $G=\mathbb{N}^{k}$, we prove that this condition is equivalent to the property that all sequences in our persistence module are of finite type (as a persistence module over $\mathbb{N}$ ), see Fig. 2, but this equivalence fails for general (good) monoids. Particularly, our second main result implies the first one, because for $G=\mathbb{N}$, the monoid ring $R[\mathbb{N}]$ is precisely the polynomial ring $R[t]$.

Outline Although our first main result is a special case of the second one, we decided to give a complete treatment of the classical case of linear sequences first. For that, we introduce the necessary basic concepts in Sect. 2 and prove the Representation Theorem in Sect. 3. The additional concepts required for the monoidal case are introduced in Sect. 4. The Generalized Representation Theorem is proved in Sect. 5. We conclude in Sect. 6.

\footnotetext{
${ }^{2}$ Recall that a monoid is almost a group, except that elements might not have inverses.
} 


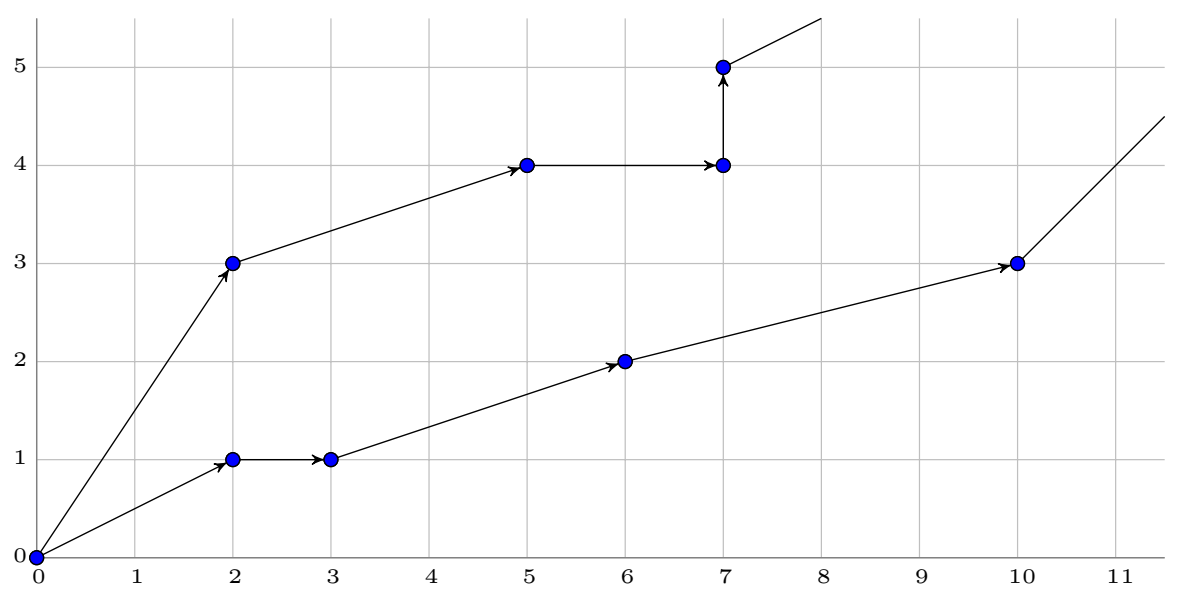

Fig. 2 Graphical illustration of two sequences in a generalized persistence module over the monoid $\mathbb{N}^{2}$. The corresponding $R$-modules and homomorphisms are not specified in the figure

\section{Basic notions}

Category theory We review some basic concepts of category theory as needed in this exposition. See Adámek et al. (2009) and Mac Lane (1971) for comprehensive introductions. A category $\mathbf{C}$ is a collection of objects and morphisms, which have to satisfy associativity and identity axioms: for all morphisms $\alpha \in \operatorname{hom}(W, X), \beta \in \operatorname{hom}(X, Y), \gamma \in \operatorname{hom}(Y, Z)$ there are compositions $\beta \circ \alpha \in \operatorname{hom}(W, Y)$ and $\gamma \circ \beta \in \operatorname{hom}(X, Z)$ which ensure $(\gamma \circ \beta) \circ \alpha=\gamma \circ(\beta \circ \alpha)$. If it is not clear which category the morphisms belong to, one writes hom ${ }_{\mathbf{C}}$ to denote the category $\mathbf{C}$. Furthermore, for all objects $X$ in a category $\mathbf{C}$ there are identity morphisms $1_{X} \in \operatorname{hom}(X, X)$ such that $1_{X} \circ \alpha=\alpha$ and $\beta \circ 1_{X}=\beta$ for all $\alpha \in \operatorname{hom}(W, X)$, $\beta \in \operatorname{hom}(X, Y)$. One example of a category is the collection of all sets as objects and maps between sets as morphisms. Others are topological spaces and continuous maps, groups and group homomorphisms, and vector spaces over a fixed field and linear maps.

A functor $F: \mathbf{C} \rightarrow \mathbf{D}$ carries the information from one category to another. It says that $F(\beta \circ \alpha)=F(\beta) \circ F(\alpha)$ and $F\left(1_{X}\right)=1_{F(X)}$ for all $\alpha \in \operatorname{hom}_{\mathbf{C}}(W, X)$, $\beta \in \operatorname{hom}_{\mathbf{C}}(X, Y)$. It suffices to define functors on the morphisms only, but usually one also specifies $F(X)$ for clarity. A simple example of a functor is the identity functor of a category, which simply maps each object and each morphism on itself. As another example, homology is a functor from the category of topological spaces to the category of abelian groups.

Functors describe similarities between two categories. Two categories $\mathbf{C}, \mathbf{D}$ are called isomorphic if there are functors $F: \mathbf{C} \rightarrow \mathbf{D}, G: \mathbf{D} \rightarrow \mathbf{C}$ such that $F \circ G$ is the identity functor on $\mathbf{D}$ and $G \circ F$ is the identity functor on $\mathbf{C}$. In this case, we also call the pair $(F, G)$ of functors an isomorphic pair.

Graded rings and modules We only consider rings with unity and usually denote them by $R$. A left-ideal $I$ of $R$ is an additive subgroup of $R$ such that $r \in R$ and $x \in I$ 
implies that $r x \in I$. Replacing $r x$ with $x r$ defines a right-ideal. A subgroup $I$ is called ideal if it is a left-ideal and a right-ideal.

An $R$-(left-)module $(M,+, \cdot)$ is an abelian group with a scalar multiplication (from the left), which is a bi-additive group action of $R$ on it. We usually denote modules by $M$. For example, every left-ideal of $R$ is also an $R$-module. An $R$-module morphism between $R$-modules $M$ and $N$ is a group homomorphism $f: M \rightarrow N$ that also satisfies $f(r x)=r f(x)$ for all $r \in R$ and $x \in M$.

A ring $S$ is $\mathbb{N}$-graded, or just graded if $S$ can be written as $S=\bigoplus_{i \in \mathbb{N}} S_{i}$ where each $S_{i}$ is an abelian group and $s_{i} \cdot s_{j} \in S_{i+j}$ whenever $s_{i} \in S_{i}$ and $s_{j} \in S_{j}$. If $R$ is a ring, the polynomial ring $R[t]$ is naturally graded with $R[t]_{i}$ being the $R$-module generated by $t^{i}$. While $R[t]$ might permit different gradings (e.g. if $R$ itself is a polynomial ring), we will always assume that $R[t]$ is graded in the above way.

If $S=\oplus S_{i}$ is a graded ring, an $S$-module $M$ is graded if there is a decomposition $M=\bigoplus_{i \in \mathbb{N}} M_{i}$ such that each $M_{i}$ is an abelian group and $s_{i} \cdot m_{j} \in M_{i+j}$ whenever $s_{i} \in S_{i}$ and $m_{j} \in M_{j}$. As a trivial example, $S$ is a graded $S$-module itself. By definition, every nonzero element of $M$ can be written as a finite sum

$$
m=m_{i_{1}}+m_{i_{2}}+\cdots+m_{i_{k}}
$$

with $k \geq 1, i_{1}<i_{2}<\cdots<i_{k}$, and $m_{i_{j}} \in M_{i_{j}}$. If $k=1, m=m_{i_{1}}$ is called homogeneous of degree $i_{1}$.

A graded morphism $f: M \rightarrow N$ between graded $S$-modules is an $S$-module morphism such that $f\left(M_{i}\right) \subset N_{i}$ for all $i \in \mathbb{N}$. Fixing a ring $R$, the collection of all graded $R[t]$-modules together with graded morphisms yields another example of a category, which we will denote by $R[t]-\mathbf{G r}-$ Mod.

Finiteness conditions for modules An $R$-module $M$ is called finitely generated if there exist finitely many elements $\mathfrak{g}_{1}, \ldots, \mathfrak{g}_{n}$ in $M$ such that every $x \in M$ can be written as $x=\sum_{i=1}^{n} \lambda_{i} \mathfrak{g}_{i}$ with $\lambda_{i} \in R$. The set $\left\{\mathfrak{g}_{1}, \ldots, \mathfrak{g}_{n}\right\}$ is called the generating set of $M$. Equivalently, $M$ is finitely generated if and only if there exists a surjective module morphism

$$
R^{n} \stackrel{\mu}{\rightarrow} M
$$

where $R^{n}$ is just the free abelian $R$-module with $n$ generators $\mathfrak{e}_{1}, \ldots, \mathfrak{e}_{n}$. If $\mu$ maps $\mathfrak{e}_{i}$ to $\mathfrak{g}_{i}$, we call $\mu$ associated to the generating set $\left\{\mathfrak{g}_{1}, \ldots, \mathfrak{g}_{n}\right\}$.

In general, $\mu$ is not injective and there are relations between the generators (which are also sometimes called syzygies). $M$ is called finitely presented if it is finitely generated and the $R$-module ker $\mu$ is finitely generated as well. Equivalently, finitely presented means that there exists an exact sequence

$$
R^{m} \rightarrow R^{n} \stackrel{\mu}{\rightarrow} M \rightarrow 0 .
$$

Clearly, finitely presented modules are finitely generated, but the converse is not true as the example from the introduction shows. Note that morphisms between finitely 
presented modules always imply morphisms on the corresponding free modules, such that the following diagram commutes:

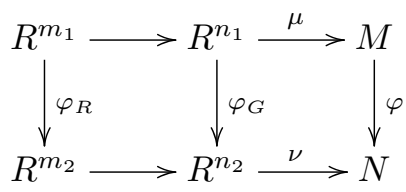

So $\varphi$ can be represented as a matrix of two blocks, which describe how generators and relations are changing. For an easy proof, see Lemma 2.1.25 in Greuel and Pfister (2007) (which also holds for non-commutative rings).

A ring $R$ is called (left-/right-)Noetherian if every (left-/right-)ideal of $R$ is finitely generated. In particular, every principal ideal domain is Noetherian. We point out the following important statements on Noetherian rings:

Lemma 1 Let $R$ be a Noetherian ring with unity. Then every finitely generated $R$-module is finitely presented. If $R$ is also commutative, then $R[t]$ is Noetherian.

Proof The second part is Hilbert's Basis Theorem (see Waerden 1993, 15.1). For the first part, see Lam (1999, Proposition 4.29).

We will mostly consider the case of finitely generated/presented graded modules. So, let $M$ be a finitely generated graded $S$-module (where $S$ is graded, but not necessarily Noetherian). It is not difficult to see that $M$ is also generated by a finite set of homogeneous elements in this case, which we will call a homogeneous generating set. With $\mu: S^{n} \rightarrow M$ associated to the homogeneous generating set $\left\{\mathfrak{g}_{1}, \ldots, \mathfrak{g}_{n}\right\}$, we define a grading on $S^{n}$ by setting $\operatorname{deg}\left(\boldsymbol{e}_{i}\right)$ as the degree of $\mathfrak{g}_{i}$ in $M$ and $\operatorname{deg}\left(s_{i}\right)=i$ for $s_{i} \in S_{i}$ in the grading $\oplus S_{i}$ of $S$. Then again, each $x \in M$ decomposes into a finite sum of elements of pairwise distinct degrees, and we can talk about homogeneous elements of $S^{n}$ accordingly. If $M$ is finitely presented, the generating set of ker $\mu$ can be chosen with homogeneous elements as well.

\section{The ZC-representation theorem}

Persistence modules and $R[t]$-modules Persistence modules are the major object of interest in the theory of persistent homology. We motivate it with the following typical example: Given a nested sequence of topological spaces indexed over the integers

$$
X_{0} \hookrightarrow X_{1} \hookrightarrow X_{2} \hookrightarrow X_{3} \hookrightarrow X_{4} \cdots
$$

then the inclusions maps $X_{i} \rightarrow X_{j}$ induce group homomorphisms $\varphi_{i, j}: H_{*}\left(X_{i}\right) \rightarrow H_{*}\left(X_{j}\right)$. By functoriality of homology, $\varphi_{i, i}$ is the identity and $\varphi_{i, j}$ is the composition of $\varphi_{k, k+1}$ for $i \leq k \leq j-1$. The following definition captures these algebraic properties: 
Definition 2 Let $R$ be a ring with unity. A discrete algebraic persistence module $(D A P M)$ is a tuple $\mathscr{M}=\left(\left(M_{i}\right)_{i \in \mathbb{N}},\left(\varphi_{i, j}\right)_{i \leq j \in \mathbb{N}}\right)$, such that $M_{i}$ is an $R$-module, $\varphi_{i, j}: M_{i} \rightarrow M_{j}$ is a module morphism, $\varphi_{i, i}=1_{M_{i}}$ and $\varphi_{i, k}=\varphi_{j, k} \circ \varphi_{i, j}$ for all $i \leq k \leq j$.

A DAPM is completely specified by the modules and the morphisms between consecutive modules, so it is usually just written as

$$
\mathscr{M}: M_{0} \stackrel{\varphi_{0}}{\longrightarrow} M_{1} \stackrel{\varphi_{1}}{\longrightarrow} \ldots \stackrel{\varphi_{i-1}}{\longrightarrow} M_{i} \stackrel{\varphi_{i}}{\longrightarrow} M_{i+1} \stackrel{\varphi_{i+1}}{\longrightarrow} \ldots
$$

where $\varphi_{i}:=\varphi_{i, i+1}$.

$D A P M s$ over $R$ are closely related to graded $R[t]$-modules: indeed, given a $D A P M$ $\mathscr{M}$ as in (1), we can associate to it a graded $R[t]$-module by setting

$$
\alpha(\mathscr{M}):=\bigoplus_{i \in \mathbb{N}} M_{i}
$$

where multiplication by $t$ is defined by $t \cdot m_{i}:=\varphi_{i}\left(m_{i}\right) \in M_{i+1}$ for $m_{i} \in M_{i}$. Vice versa, an $R[t]$-module $\bigoplus_{i \in \mathbb{N}} M_{i}$ defines a $D A P M$ by

$$
\beta\left(\bigoplus_{i \in \mathbb{N}} M_{i}\right):=M_{0} \stackrel{\varphi_{0}}{\longrightarrow} M_{1} \stackrel{\varphi_{1}}{\longrightarrow} M_{2} \rightarrow \cdots
$$

where the morphisms are just multiplication with $t$, that is $\varphi_{i}\left(m_{i}\right):=t \cdot m_{i}$.

Definition 3 For two DAPMs $\left(\left(M_{i}\right)_{i \in \mathbb{N}},\left(\varphi_{i, j}\right)_{i \leq j \in \mathbb{N}}\right),\left(\left(N_{i}\right)_{i \in \mathbb{N}},\left(\psi_{i, j}\right)_{i \leq j \in \mathbb{N}}\right)$, a family $\xi_{*}=\left(\xi_{i}: M_{i} \rightarrow N_{i}\right)_{i \in \mathbb{N}}$ of module morphisms is called discrete algebraic persistence module morphism if $\psi_{i, j} \circ \xi_{i}=\xi_{j} \circ \varphi_{i, j}$. Equivalently, the following diagram commutes:

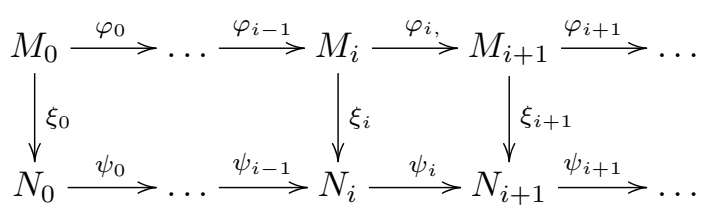

With such morphisms, the class of all DAPMs over $R$ becomes a category, which we call $R$-Persmod.

Lemma 4 The maps $\alpha$ and $\beta$ from (2) and (3) extend to functors between $R$-Persmod and $R[t]-\mathbf{G r}-$ Mod which form an isomorphic pair of functors. In particular, the two categories are isomorphic.

Proof Given a DAPM morphism 


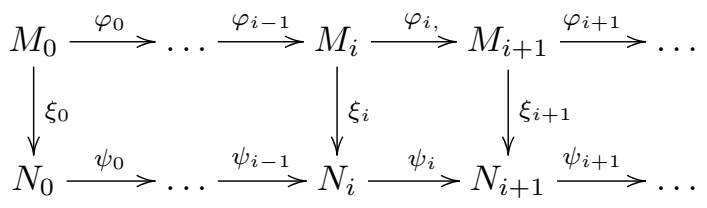

between two DAPMs $\mathscr{M}$ and $\mathcal{N}$, we define

$$
\alpha\left(\xi_{*}\right): \bigoplus_{i \in \mathbb{N}} M_{i} \rightarrow \bigoplus_{i \in \mathbb{N}} N_{i},\left(m_{i}\right)_{i \in \mathbb{N}} \mapsto\left(\xi_{i}\left(m_{i}\right)\right)_{i \in \mathbb{N}}
$$

It is straight-forward to check that $\alpha\left(\xi_{*}\right)$ is a well-defined morphism between $\alpha(\mathscr{M})$ and $\alpha(\mathcal{N})$ and that $\alpha$ has the functorial properties-see Appendix 1.

Vice versa, a morphism

$$
\eta: \bigoplus_{i \in \mathbb{N}} M_{i} \rightarrow \bigoplus_{i \in \mathbb{N}} N_{i}
$$

in $R[t]$-Gr - Mod induces a homomorphism $\eta_{i}: M_{i} \rightarrow N_{i}$ for each $i \in \mathbb{N}$, and these induced maps are compatible with multiplication with $t$. Hence, the diagram

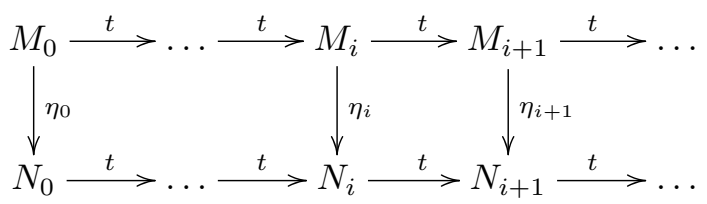

commutes and so, setting $\beta(\eta):=\left(\eta_{0}, \eta_{1}, \ldots\right)$ yields a $D A P M$ morphism between $\beta\left(\oplus_{i \in \mathbb{N}} M_{i}\right)$ and $\beta\left(\bigoplus_{i \in \mathbb{N}} N_{i}\right)$. Again, we defer the proof of functoriality of $\beta$ to Appendix 1.

Finally, the construction immediately implies that $\alpha \circ \beta$ equals the identity functor on $R[t]-\mathbf{G r}-$ Mod and $\beta$ o $\alpha$ equals the identity functor on $R$-Persmod.

Finiteness conditions In the context of computation and classification, it is natural to impose some finiteness condition on persistence modules, yielding a full subcategory of $R$-Persmod. Restricting the functor $\alpha$ from Lemma 4 to this subcategory yields a corresponding subcategory of $R[t]$-Gr - Mod. But does the correspondence established above also carry over the finiteness condition in an appropriate way? This is the question we study in this subsection.

Definition 5 A $D A P M \mathscr{M}=\left(\left(M_{i}\right)_{i \in \mathbb{N}},\left(\varphi_{i, j}\right)_{i \leq j \in \mathbb{N}}\right)$ is of finite type if there is a $D \in \mathbb{N}$ such that for all $D \leq i \leq j$ the map $\varphi_{i, j}$ is an isomorphism. $\mathscr{M}$ is called of finitely 
presented (generated) type if it is of finite type and $M_{i}$ is finitely presented (generated) as an $R$-module for all $i \in \mathbb{N}$.

We will show next that DAPMs of finitely presented type over $R$ are isomorphic to finitely presented graded $R[t]$-modules using the functors $\alpha$ and $\beta$ above.

Lemma 6 If a DAPM $\mathscr{M}=\left(\left(M_{i}\right)_{i \in \mathbb{N}},\left(\varphi_{i, j}\right)_{i \leq j \in \mathbb{N}}\right)$ is of finitely presented type, $\alpha(\mathscr{M})$ is finitely presented.

Proof Let $D \in \mathbb{N}$ be such that for all $D \leq i \leq j, \varphi_{i, j}: M_{i} \rightarrow M_{j}$ is an isomorphism. Let $\mathfrak{G}_{i}$ be a generating set for $M_{i}$. We claim that $\bigcup_{i=1}^{D^{D}} \mathfrak{G}_{i}$ is a generating set for $\alpha(\mathscr{M})$. To see that, it suffices to show that every homogeneous element in $\alpha(\mathscr{M})=\oplus M_{i}$ is generated by the union of the $G_{i}$. So, fix $k \in \mathbb{N}$ and $m_{k}$ homogeneous of degree $k$. If $k \leq D$, then $m_{k}$ is generated by the elements of $\mathfrak{G}_{k}$ by construction. If $k>D$, we show that $m_{k}$ is generated by $\mathfrak{G}_{D}$. For that, let $m_{D}:=\varphi_{D, k}^{-1}\left(m_{k}\right)$ which exists because $\varphi_{D, k}$ is isomorphism. $m_{D}$ is generated by $\mathfrak{G}_{D}$, hence $m_{k}$ is generated by $\varphi_{D, k}\left(\mathfrak{G}_{D}\right)$. By construction of $\alpha, \varphi_{D, k}\left(\mathfrak{G}_{D}\right)=t^{k-D} \mathfrak{G}_{D}$ and since $t^{k-D}$ is a ring element in $R[t], m_{k}$ is generated by $\mathfrak{G}_{D}$. This shows that $\alpha(\mathscr{M})$ is finitely generated.

It remains to show that $\alpha(\mathscr{M})$ is also finitely presented. Let $\mu_{i}: R^{n_{i}} \rightarrow M_{i}$ be the generating surjective map that corresponds to $\mathfrak{G}_{i}$. Writing $n=\sum_{i=1}^{D} n_{i}$, there is a map $\mu: R[t]^{n} \rightarrow \alpha(\mathscr{M})$ that corresponds to the generating set $\bigcup_{i=1}^{D} \mathfrak{G}_{i}$. If $\mathfrak{g}_{i}$ is a generator, we will use the notation $\mathrm{e}_{i}$ to denote the corresponding generator of $R[t]^{n}$.

We now define a finite set of elements of ker $\mu$. First of all, let $\boldsymbol{Z}_{i}$ be the generating set of ker $\mu_{i}$ for $0 \leq i \leq D$. Clearly, all elements of $\boldsymbol{Z}_{i}$ are also in ker $\mu$. Moreover, for any $0 \leq i<j \leq D$, and any generator $\mathfrak{g}_{i}$ in $\mathfrak{G}_{i}$ with $\varphi_{i, j}\left(\mathfrak{g}_{i}\right) \neq 0$, we can write

$$
\varphi_{i, j}\left(\mathfrak{g}_{i}\right)=\sum_{v=0}^{n_{j}} \lambda_{\nu} \mathfrak{g}_{j}^{(v)}
$$

where $\lambda_{v} \in R$ and $\mathfrak{G}_{j}=\left\{\mathfrak{g}_{j}^{(0)}, \ldots, \mathfrak{g}_{j}^{\left(n_{j}\right)}\right\}$. In that case, the corresponding element

$$
t^{j-i} \boldsymbol{e}_{i}-\sum_{v=0}^{n_{j}} \lambda_{v} \mathbf{e}_{j}^{(v)}
$$

is in ker $\mu$. We let $\boldsymbol{Z}_{i, j}$ denote the (finite) set obtained by picking one element as above for each $\mathfrak{g}_{i}$ with $\varphi_{i, j}\left(\mathfrak{g}_{i}\right) \neq 0$. We claim that $\boldsymbol{\beta}:=\bigcup_{i=0}^{D} \boldsymbol{Z}_{i} \cup \bigcup_{0 \leq i<j \leq D} \boldsymbol{B}_{i, j}$ generates ker $\mu$ :

Fix an element in $x \in \operatorname{ker} \mu$, which is of the form

$$
x=\sum_{\ell} \lambda_{\ell} e_{\ell}
$$


with $\lambda_{\ell} \in R[t]$ and $\boldsymbol{e}_{\ell}$ a generator of $R[t]^{n}$. We can assume that $x$ is homogeneous of some degree $k$. We first consider the case that $k \leq D$ and all $\lambda_{\ell}$ are of degree 0 . Then, all $\mathfrak{e}_{\ell}$ that appear in $x$ are of the same degree, and hence, their images under $\mu$ are generators of $M_{k}$. It follows that $x$ is generated by the set $\boldsymbol{Z}_{k}$.

Next, we consider the case that $k \leq D$, and some $\lambda_{\ell}$ is of positive degree. Because $x$ is homogeneous, $\lambda_{\ell}$ is then of the form $r_{\ell} t^{d_{\ell}}$ for some $r_{\ell} \in R$ and $d_{\ell}>0$. Since the degree of $\boldsymbol{e}_{\ell}$ is $k-d_{\ell}$, there is an element $\boldsymbol{z}_{\ell}$ in $\boldsymbol{Z}_{k-d_{\ell}, k}$ of the form $\boldsymbol{z}_{\ell}=t^{d_{\ell}} \mathbf{e}_{\ell}-\sum_{v=0}^{n_{\ell}} \tilde{\lambda}_{v} \mathbf{e}_{k}^{(v)}$ with all $\mathfrak{e}_{k}^{(v)}$ of degree $k$ and each $\tilde{\lambda}_{v} \in R$. Then in $x-r_{\ell} \boldsymbol{z}_{\ell}$ the coefficient of $\boldsymbol{e}_{\ell}$ in $x$ is 0 , and we only introduce summands with coefficients of degree 0 in $t$.

Iterating this construction for each summand with coefficient of positive degree, we get an element $x^{\prime}=x-\sum_{w} r_{w} \boldsymbol{z}_{w}$ with $r_{w} \in R$ and $\boldsymbol{z}_{w}$ elements of $\mathfrak{Z}$, and $x^{\prime}$ only having coefficient of degree 0 in $t$. This yields to $x=x^{\prime}+\sum_{w} r_{w} z_{w}$. Using the first part, it follows that $x$ is generated by $\mathbb{Z}$.

Finally, we consider the case that $k>D$. In that case, each $\lambda_{\ell}$ is of degree at least $k-D$, because the maximal degree of $\mathfrak{e}_{\ell}$ is $D$. So, $x=t^{k-D} x^{\prime}$ with $x^{\prime}$ homogeneous of degree $D$. Since $0=\mu(x)=t^{k-D} \mu\left(x^{\prime}\right), x^{\prime} \in$ ker $\mu$ as well. By the second part, $x^{\prime}$ is hence generated by $\mathbb{Z}$, and so is $x$.

For the next two lemmas, we fix a finitely presented graded $R[t]$-module $\mathbf{M}:=\bigoplus_{i \in \mathbb{N}} M_{i}$ with a map

$$
R[t]^{n} \stackrel{\mu}{\rightarrow} \mathbf{M}
$$

such that ker $\mu$ is finitely generated. Moreover, we let $\mathfrak{G}:=\left\{\mathfrak{g}^{(1)}, \ldots, \mathfrak{g}^{(n)}\right\}$ denote generators of $\mathbf{M}$ and $\mathfrak{Z}:=\left\{\mathfrak{z}^{(1)}, \ldots, \mathfrak{z}^{(m)}\right\}$ denote a generating set of ker $\mu$. We assume that each $\mathfrak{g}^{(i)}$ and each $\mathfrak{z}^{(j)}$ is homogeneous (with respect to the grading of the corresponding module), and we let $\operatorname{deg}\left(\mathfrak{g}^{(i)}\right), \operatorname{deg}\left(\mathfrak{z}^{(j)}\right)$ denote the degrees. We further assume that $\mathfrak{G}$ and $\mathbb{Z}$ are sorted by degrees in non-decreasing order.

Lemma 7 Each $M_{i}$ is finitely presented as an R-module.

Proof We argue first that $M_{i}$ is finitely generated. Set $d_{j}:=\operatorname{deg}\left(\mathfrak{g}^{(j)}\right)$ for $1 \leq j \leq n$. Let $n_{i}$ denote the number of elements in $\mathfrak{G}$ with degree at most $i$. Define the map $\mu_{i}: R^{n_{i}} \rightarrow M_{i}$, by mapping the $j$ th generator $\mathrm{e}_{i}^{(j)}$ of $R^{n_{i}}$ to the element $t^{i-d_{j}} \mathfrak{g}^{(j)}$. It is then straight-forward to see that the map $\mu_{i}$ is surjective, proving that $M_{i}$ is finitely generated.

We show that ker $\mu_{i}$ is finitely generated as well. Let $\mathrm{e}^{(1)}, \ldots, \mathrm{e}^{(n)}$ be the generators of $R[t]^{n}$ mapping to $\mathfrak{g}^{(1)}, \ldots, \mathfrak{g}^{(n)}$ under $\mu$. Let $m_{i}$ denote the number of elements in $\mathbf{Z}$ such that $d_{j}^{\prime}:=\operatorname{deg}\left(\mathfrak{z}^{(j)}\right) \leq i$. For every $\mathfrak{z}^{(j)}$ with $1 \leq j \leq m_{i}$, consider $t^{i-d_{j}^{\prime}} \mathfrak{z}^{(j)}$, which

can be written as

$$
t^{i-d_{j}^{\prime}} \boldsymbol{z}^{(j)}=\sum_{k=1}^{n_{i}} r_{k} t^{i-d_{k}} \mathbf{e}^{(k)}
$$


with $r_{k} \in R$. Now, define

$$
\mathfrak{\jmath}_{i}^{(j)}:=\sum_{k=1}^{n_{i}} r_{k} \mathbf{e}_{i}^{(k)}
$$

and define $\boldsymbol{Z}_{i}:=\left\{\boldsymbol{z}_{i}^{(j)} \mid 1 \leq i \leq m_{i}\right\}$. We claim that $\boldsymbol{Z}_{i}$ generates ker $\mu_{i}$. First of all, it is clear that $\mu_{i}\left(z_{i}^{(j)}\right)=\mu\left(z^{(j)}\right)=0$. Now fix $x \in$ ker $\mu_{i}$ arbitrarily. Then, $x$ is a linear combination of elements in $\left\{\boldsymbol{e}_{i}^{(1)}, \ldots, \boldsymbol{e}_{i}^{\left(n_{i}\right)}\right\}$ with coefficients in $R$. Replacing $\mathbf{e}_{i}^{(j)}$ with $t^{i-d_{j}} \mathbf{e}^{(j)}$, we obtain $x^{\prime} \in R[t]^{n}$ homogeneous of degree $i$. By assumption, we can write $x^{\prime}$ as linear combination of elements in $\mathbb{Z}$, that is,

$$
x^{\prime}=\sum_{k=1}^{m_{i}} r_{k}^{\prime} t^{i-d_{k}^{\prime}} \mathfrak{z}^{(k)}
$$

with $r_{k}^{\prime} \in R$. Then, it holds that

$$
x=\sum_{k=1}^{m_{i}} r_{k}^{\prime} \mathfrak{z}_{i}^{(k)},
$$

which follows simply by comparing coefficients: let $j \in\left\{1, \ldots, n_{i}\right\}$ and let $c_{j} \in R$ be the coefficient of $\mathbf{e}_{i}^{(j)}$ in $x$. Let $c_{j}^{\prime}$ be the coefficient of $\mathbf{e}_{i}^{(j)}$ in the sum $\sum_{k=1}^{m_{i}} r_{k}^{\prime} \mathfrak{z}_{i}^{(k)}$, expanding each $\mathfrak{z}_{i}^{(k)}$ by its linear combination as above. Then by construction, $c_{j}$ is the coefficient of $t^{i-d_{j}} \mathbf{e}^{(j)}$ in $x^{\prime}$, and $c_{j}^{\prime}$ is the coefficient of $t^{i-d_{j}} \mathbf{e}^{(j)}$ in the sum $\sum_{k=1}^{m_{i}} r_{k}^{\prime} t^{i-d_{k}^{\prime}} \mathfrak{z}^{(k)}$. Since this sum equals $x^{\prime}$, it follows that $c_{j}=c_{j}^{\prime}$. Since $x$ was chosen arbitrary from ker $\mu_{i}$, it follows $\boldsymbol{Z}_{i}$ generates the kernel.

Lemma $8 \beta(\mathbf{M})$ is of finite type. In particular, it is of finitely presented type with Lemma 7.

\section{Proof Fix}

$$
D:=\max \left\{\operatorname{deg}\left(\mathfrak{g}^{(j)}\right), \operatorname{deg}\left(\mathfrak{z}^{(k)}\right) \mid 1 \leq j \leq n, 1 \leq k \leq m\right\} .
$$

It suffices to show that multiplication by $t$ induces an isomorphism $M_{i} \rightarrow M_{i+1}$ for every $i \geq D$. Let $y \in M_{i+1}$. Then, $y=\sum_{j=1}^{n} \lambda_{j} \mathfrak{g}^{(j)}$ with $\lambda_{j} \in R[t]$ of degree at least 1 . 
Hence, $y=t y^{\prime}$ with $y^{\prime} \in M_{i}$, showing that multiplication with $t$ gives a surjective map.

For injectivity, let $y \in M_{i}$ such that $t y=0$. Let $x \in R[t]^{n}$ be such that $\mu(x)=y$. Then $\mu(t x)=t y=0$. Hence, $t x$ can be written as

$$
t x=\sum_{j=0}^{m} \tilde{\lambda}_{j} \mathfrak{z}^{(j)}
$$

where each non-trivial $\lambda_{j}$ is a polynomial of degree at least one, because each $\mathfrak{z}^{(j)}$ is of degree at most $D$ and $t x$ is of degree at least $D+1$. Therefore, there is also a decomposition

$$
t x=\sum_{j=0}^{m} t \lambda_{j} \mathfrak{z}^{(j)}=t \sum_{j=0}^{m} \lambda_{j} \mathfrak{z}^{(j)} .
$$

Since $R[t]^{n}$ is free, this implies that $x$ equals the sum on the right hand side, implying that $x \in \operatorname{ker} \mu$, so $y=0$.

The Representation Theorem The preceding lemmas of this section immediately reply the following version of the Representation Theorem.

Theorem 9 Let $R$ be a ring with unity. The category of finitely presented graded $R[t]$-modules is isomorphic to the category of discrete algebraic persistence modules of finitely presented type.

Proof The two categories are subcategories of $R[t]-\mathbf{G r}$ - Mod and $R$-Persmod, respectively. Since $\alpha$ and $\beta$, restricted to these subcategories, map a DAPM of finitely presented type to a finitely presented graded $R[t]$-module (Lemma 6 ) and vice versa (Lemmas 7 and 8), these categories are isomorphic.

What happens if we replace "finitely presented" with the weaker condition "finitely generated" throughout? The proof of Lemma 6 shows that if $\mathscr{M}$ is of finitely generated type, $\alpha(\mathscr{M})$ is finitely generated. Vice versa, if a graded $R[t]$-module $\mathbf{M}=\bigoplus_{i \in \mathbb{N}} M_{i}$ is finitely generated, each $M_{i}$ is finitely generated, too. However, it does not follow in general that $\beta(\mathbf{M})$ is of finite type, as the example from the introduction shows. This problem disappears with additional requirements on the ring:

Corollary 10 If $R$ is commutative and Noetherian, the category of finitely generated graded $R[t]$-modules is isomorphic to the category of discrete algebraic persistence modules of finitely generated type.

Proof By Lemma 1, the corollary is just Theorem 9 restated. 
In Appendix 3, we sketch an alternative proof of this statement using Artin-Rees theory.

\section{Preliminaries on monoid structures}

A monoid is a set $G$ with a binary associative operation $\star$ and a neutral element $e$, i.e., for all $g_{1}, g_{2}, g_{3} \in G$ we have $g_{1} \star g_{2} \in G,\left(g_{1} \star g_{2}\right) \star g_{3}=g_{1} \star\left(g_{2} \star g_{3}\right)$ and $e \star g_{1}=g_{1} \star e=g_{1}$. A monoid is called commutative if $g_{1} \star g_{2}=g_{2} \star g_{1}$ for all $g_{1}, g_{2} \in G$. We usually denote a monoid by $G$ or $(G, \star)$. We sometimes omit denoting $\star$ if it clarifies the expression and no confusion is possible.

Monoid rings and gradings Let $(G, \star)$ be a monoid and $R$ be a ring with unity. The monoid ring $R[G]$ is defined as free $R$-module with basis $\left\{X^{h}\right\}_{h \in H}$ where ring multiplication of $R[G]$ is induced by its scalar multiplication as $R$-module and $X^{h_{1}} X^{h_{2}}:=X^{h_{1} \star h_{2}}$. It can be easily verified that with these operations $R[G]$ indeed becomes a ring with unity $X^{e}$. Note that both associativity of $\star$ and the existence of a neutral element in $G$ are required to guarantee a ring structure with unity on $R[G]$. Moreover, $R[G]$ is commutative if and only if both $R$ and $G$ are commutative. Furthermore, one can easily verify that $a X^{g}=X^{g} a$ in $R[G]$ whenever $a \in R$ and $g \in G$.

Monoid rings are a generalization of polynomial rings. For instance, with $R$ a ring, every $a \in R[t]$ has the form $\sum_{n \in \mathbb{N}} a_{n} t^{n}$ where almost all $a_{n}=0$. By canonical identification of $t^{n}$ with $X^{n} \in R[\mathbb{N}]$, the two notions are isomorphic. Completely analogously, one can obtain $R\left[\mathbb{N}^{n}\right] \cong R\left[t_{1}, \ldots, t_{n}\right]$ for all $0 \neq n \in \mathbb{N}$. In general, we will often write elements of $R[G]$ in the form $\sum_{g \in G} a_{g} g$ with $a_{g} \in R$ and almost all $a_{g}=0$.

The concept of gradings extends from natural numbers to arbitrary monoids without problems: a ring $S$ is called $G$-graded ring if $S=\bigoplus_{g \in G} S_{g}$ as abelian groups and $S_{g_{1}} S_{g_{2}} \subseteq S_{g_{1} \star g_{2}}$ for all $g_{1}, g_{2} \in G$. Monoid rings $R[G]$ are typical examples for $G$-graded rings. For a $G$-graded ring $S$, an $S$-module $M$ is called $G$-graded module if $M=\bigoplus_{g \in G} M_{g}$ as abelian groups and $S_{g_{1}} M_{g_{2}} \subseteq M_{g_{1} \star g_{2}}$ for all $g_{1}, g_{2} \in G$. A $G$-graded module morphism $f: M \rightarrow N$ between $G$-graded $S$-modules is a module morphism with $f\left(M_{g}\right) \subset N_{g}$ for all $g \in G$. Equivalently, one can think of $f$ as a family $\left(f_{g}\right)_{g \in G}$

of morphisms on the components with $f_{g}: M_{g} \rightarrow N_{g}$ for all $g \in G$. A $G$-graded module morphism is an isomorphism if each $f_{g}$ is an isomorphism. Given a ring $R$ and a monoid $G$, we denote the category of all $G$-graded modules over $R[G]$ and $G$-graded morphisms between $G$-graded $R[G]$-modules by $R[G]-\mathbf{G r}$ - Mod.

Good monoids The categories $R[G]-\mathbf{G r}$ - Mod exist without further assumptions on monoids. However, in order to generalize the Representation Theorem to monoids, we will require a few additional properties on monoids.

A monoid is called right-cancellative if $g_{1} \star g_{3}=g_{2} \star g_{3}$ implies $g_{1}=g_{2}$. Similarly, it is called left-cancellative if $g_{1} \star g_{2}=g_{1} \star g_{3}$ implies $g_{2}=g_{3}$. It is called cancellative if it is right-cancellative and left-cancellative. For commutative monoids these three properties are equivalent and in this case we simply call such a monoid cancellative. A (left-/right-)cancellative monoid $G$ admits (left-/right-) 


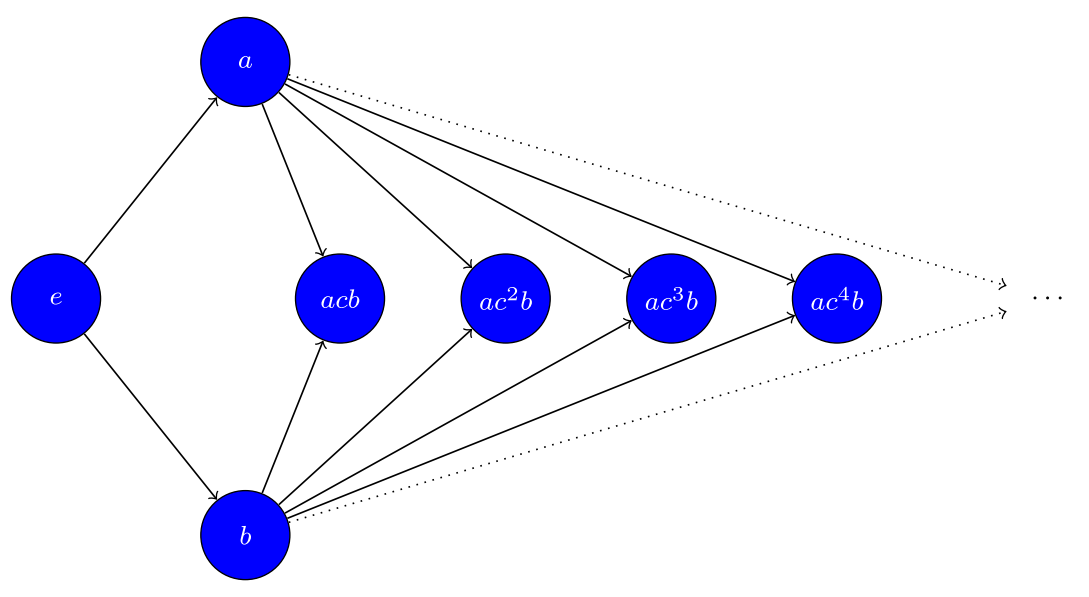

Fig. 3 Graphical illustration of a monoid that is not good. The monoid is the non-commutative monoid generated by three elements $a, b, c$ modulo the congruence generated by $a c^{n} b \approx b c^{n} a$ for all $n \in \mathbb{N}_{>0}$. The plcm of $\{a, b\}$ are the elements of the countable set $\left\{a c^{n} b\right\}_{n \in \mathbb{N}_{>0}}$

cancellativity on a monoid ring $R[G]$ with respect to multiplication by a monoid element (from the left/right).

Examples of non-cancellative monoids are $[0,1]$ with multiplication, matrices with multiplication and $\left(\mathbb{Z}_{2}, \mathbb{N} \cup\{\infty\}\right)$ where $(x, n) \star(y, m):=(x y, \min (n, m))$ and $e=(0, \infty)$. An example for a right-cancellative monoid that is not left-cancellative is the monoid $\left\{e, g_{1}, g_{2}, g_{3}\right\}$ where $e$ is the neutral element and $g_{i} \star g_{j}:=g_{i}$ for all $i \in\{1,2,3\}$.

We call $g_{2}$ a (left-)multiple of $g_{1}$ and write $g_{1} \preceq g_{2}$ if there exists an $h \in G$ such that $h \star g_{1}=g_{2}$. A proper multiple of $g_{1}$ is a multiple $g_{2}$ with $g_{1} \neq g_{2}$, written as $g_{1} \prec g_{2}$. For a subset $\tilde{G} \subseteq G$ an element $h$ is called common multiple of $\tilde{G}$ if $g \preceq h$ for all $g \in \tilde{G}$. We call a common multiple $h$ of $\tilde{G}$ partially least, if there is no multiple $h^{\prime}$ of $\tilde{G}$ such that $h^{\prime} \prec h$. We write plcm for partially least common multiples. We say that the monoid $G$ is weak plcm if for any finite subset $H \subseteq G$ there are at most finitely many distinct partially least common multiples of $\mathrm{H}^{3}$

A monoid is anti-symmetric if $g_{1} \preceq g_{2}$ and $g_{2} \preceq g_{1}$ imply that $g_{1}=g_{2}$. This is equivalent to the condition that $\preceq$ turns $G$ into a poset. In an anti-symmetric monoid, no element (except $e$ ) can have an inverse element.

Definition 11 We call a monoid good if it is cancellative, anti-symmetric and weak plcm.

Some easy commutative examples for good monoids with uniquely existing plcms are $\left(\mathbb{N}^{k},+\right),\left(\mathbb{Q}_{\geq 0}^{k},+\right),\left(\mathbb{R}_{\geq 0}^{k},+\right),((0,1], \cdot),(\mathbb{Q} \cap(0,1], \cdot)$ and $([1, \infty), \cdot)$. A fundamental class of good monoids are free monoids, which can be expressed as finite

\footnotetext{
${ }^{3}$ It is called "weak" plcm to point out that the plcm does not have to exist or to be unique.
} 
sequences of elements of a set. In non-commutative free monoids, a subset admits common left multiples if and only if it contains a maximal element. If this exists, it is also the unique plcm. Constructing cancellative anti-symmetric monoids that are not weak plcm does not come naturally. Consider the non-commutative free monoid generated by $a, b, c$. Introduce the congruence generated by $a c^{n} b \approx b c^{n} a$ for all $n \in \mathbb{N}_{>0}$ (see Fig. 3). Then there are infinitely many plcm for $\{a, b\}$ in the quotient monoid, since every equivalence class with representative $a c^{n} b$ is partially least for $\{a, b\}$. In particular, good monoids are not closed under homomorphisms.

A convenient way to visualize a monoid is a directed multi-graph where each vertex corresponds to an element of $G$. For a vertex corresponding to $g \in G$, there is an outgoing edge for each $h \in G$ going to the vertex $h \star g$. We ignore the self loops induced by the neutral element of $G$ on each vertex. In this interpretation, $g_{1} \preceq g_{2}$ if and only if there is an edge from the vertex labeled $g_{1}$ to the vertex labeled $g_{2}$ in the graph. Rightcancellativity means that there is at most one edge between any pair of vertices, that is, the multi-graph is a graph. Left-cancellativity means that edges obtained by multiplication by $h$ from pairwise distinct vertices $g_{1}, \ldots, g_{n}$ can not lead to the same vertex $g$. Anti-symmetry simply implies that the graph is acyclic (modulo self-loops). Weak plcm means that each finite subset of vertices has a finite set of minimal common successors. As a consequence, a good monoid is either trivial or infinite, because if $G$ has at least two elements, there are at least two outgoing edges per vertex, and one of them cannot be a self-loop because of right-cancellativity. Because of acyclicity, we can thus form an infinite sequence of elements. For concrete pictures, it is usually convenient to draw only a subset of vertices and edges.

Related concepts The cancellation property is a classical assumption (Clifford and Preston 1961) and is sometimes even part of the definition of a monoid (Geroldinger and Halter-Koch 2006). The property of a monoid being weak plcm is less standard. It gives rise to a connection to the vivid branch of factorization theory (Baeth and Smertnig 2015; Geroldinger and Halter-Koch 2006). Least common multiples are defined in a related way, namely that a (left-)lcm of a set of elements of a monoid is a right-divisor of all other common multiples. If a monoid is cancellative and anti-symmetric, then any set of lcms consists of at most one element. Therefore, monoids with these assumptions or with uniquely existing $\mathrm{lcms}$ are sometimes called $\mathrm{lcm}$ monoids (Dehornoy 2000). Note that the existence of a plcm does not imply the existence of an lcm. Conversely, if an lcm exists and is unique, it is also the unique plcm. Therefore, divisibility monoids (Kuske 2001) Garside monoids (Dehornoy et al. 2015) and Gaussian monoids (Dehornoy and Paris 1999) are examples for subclasses of good monoids. These subclasses are involved in trace theory (Droste and Kuske 2001) and braid theory (Dehornoy 2000; Dehornoy and Paris 1999; Dehornoy et al. 2015), respectively. See Fig. 4 for a concrete example of a Garside monoid. Anti-symmetric monoids are sometimes also called centerless, conical, positive, zerosumfree (Wehrung 1996), reduced (Geroldinger and Halter-Koch 2006) or monoids with trivial unit group Clifford and Preston (1961). The preorder induced by left-factorization is related to one of Green's preorders (Grillet 2017). Given an anti-symmetric monoid $G$, the aforementioned preorder is a partial order and gives rise to a right-partially ordered monoid $(G, \star, \preceq)$, since clearly $g_{1} \preceq g_{2}$ implies $h \star g_{1} \preceq h \star g_{2}$ for all $h \in G$. If $G$ is commutative, then $(G, \star, \preceq)$ is a partially ordered monoid. A formulation of the interleaved equivalence of persistence modules 


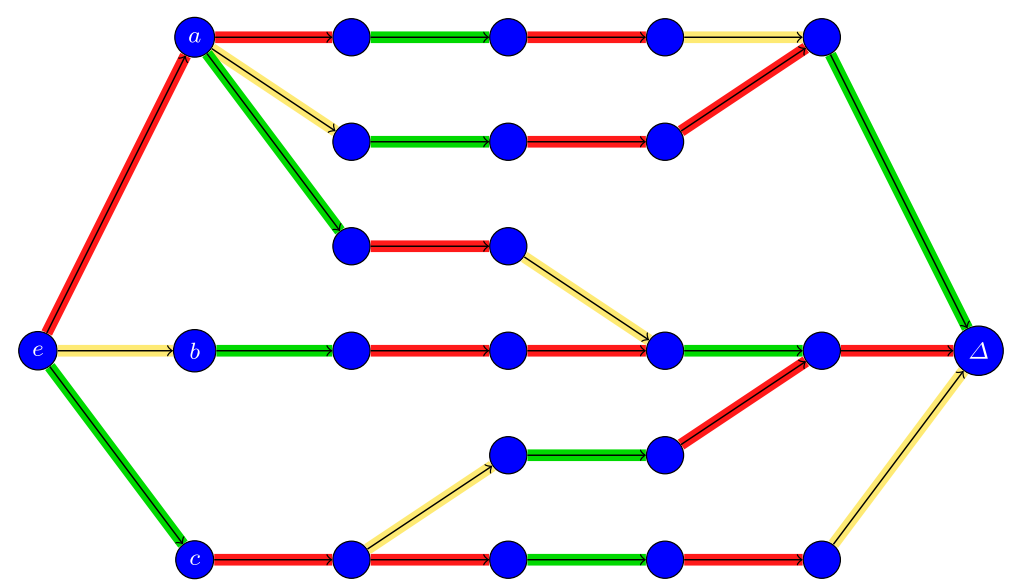

Fig. 4 Hasse diagram illustrating a finite part of a good monoid. It is the Garside monoid obtained by the free non-commutative monoid $\langle a, b, c\rangle$ modulo the congruence generated by $b a c a \approx a^{2} c b, c a^{2} c b \approx a c b a c$ and $a c b a c \approx c b a c a$. The redly, yellowly and greenly highlighted arrows represent left-multiplication with the elements $a, b$ and $c$, respectively. A Garside element of a monoid is an element whose left and right divisors coincide, are finite and generate the monoid. $\Delta$ is the minimal Garside element. For more details on this example and how such examples can be considered as Garside monoids or divisibility monoids we refer to Picantin (2005) (color figure online)

over the totally ordered monoids $\mathbb{N}$ and $\mathbb{R}$ can be found in Vejdemo-Johansson (2012). Interleaving metrics can even be defined for functors from a fixed preordered set to a fixed arbitrary category (Bubenik et al. 2015).

\section{The representation theorem over monoids}

Generalized persistence modules and $R[G]$-modules We will now extend the Representation Theorem from linear sequences of the form

$$
M_{0} \stackrel{\varphi_{0}}{\longrightarrow} M_{1} \stackrel{\varphi_{1}}{\longrightarrow} M_{2} \stackrel{\varphi_{2}}{\longrightarrow} \ldots
$$

to representations of a monoid $(G, \star, \preceq)$, right-partially ordered by left factorization. For simplicity, we will assume throughout this section that $(G, \star)$ is a good monoid-see the conclusion for a discussion of how the conditions of $G$ could be further relaxed. We define persistence modules over $G$ :

Definition 12 Let $R$ be a ring with unity. A generalized algebraic persistence module $(G A P M)$ is a tuple $\mathscr{M}_{G}=\left(\left(M_{g}\right)_{g \in G},\left(\varphi_{g_{1}, g_{2}}\right)_{g_{1} \leq g_{2} \in G}\right)$ such that $M_{g}$ is an $R$-module, $\varphi_{g_{1}, g_{2}}: M_{g_{1}} \rightarrow M_{g_{2}}$ is a module morphism, $\varphi_{g, g}=1_{M_{g}}$ and $\varphi_{g_{1}, g_{3}} \stackrel{g}{=} \varphi_{g_{2, g_{3}}} \circ \varphi_{g_{1}, g_{2}}$ for

all $g \in G, g_{1} \preceq g_{2} \preceq g_{3} \in G$. 
Much more succinctly, we could equivalently define a $G A P M$ as a functor from the poset category $G$ to $R$ - Mod. It is clear that a $G A P M$ over the monoid $\mathbb{N}$ is just a DAPM. Of interest is also the case $G=\left(\mathbb{N}^{k},+\right)$, which has been investigated for example in Carlsson and Zomorodian (2009).

As in the case of $G=\mathbb{N}$, arbitrary $G A P M$ are closely related to $R[G]$-modules. Given a $G A P M \mathscr{M}_{G}$, we can assign a graded $R[G]$-module to it by setting

$$
\alpha\left(\mathscr{M}_{G}\right):=\bigoplus_{g \in G} M_{g}
$$

where multiplication by an element $h \in G$ is defined by $h \cdot m_{g}:=\varphi_{g, h \star g}\left(m_{g}\right) \in M_{h \star g}$ for all $g \in G$ and all $m_{g} \in M_{g}$.

Vice versa, an $R[G]$-module $\oplus_{g \in G} M_{g}$ defines a $G A P M$ by

$$
\beta\left(\bigoplus_{g \in G} M_{g}\right):=\left(\left(M_{g}\right)_{g \in G},\left(\varphi_{g_{1}, g_{2}}\right)_{g_{1} \leq g_{2} \in G}\right)
$$

where the morphisms are again defined conversely. More precisely, for all $g_{1} \preceq g_{2} \in G$ and all $m_{g_{1}} \in M_{g_{1}}$, we define $\varphi_{g_{1}, g_{2}}\left(m_{g_{1}}\right):=h \cdot m_{g_{1}}$ with $h \star g_{1}=g_{2}$. Note that $h$ is uniquely defined because $G$ is assumed to be right-cancellative.

Definition 13 A family of module morphisms $\xi_{G}=\left(\xi_{g}: M_{g} \rightarrow N_{g}\right)_{g \in G}$ between two $G A P M\left(\left(M_{g}\right)_{g \in G},\left(\varphi_{g_{1}, g_{2}}\right)_{g_{1} \leq g_{2} \in G}\right),\left(\left(N_{g}\right)_{g \in G},\left(\psi_{g_{1}, g_{2}}\right)_{g_{1} \leq g_{2} \in G}\right)$ over the same ring $R$ with unity is called generalized algebraic persistence module morphism if

$$
\psi_{g_{1}, g_{2}} \circ \xi_{g_{1}}=\xi_{g_{2}} \circ \varphi_{g_{1}, g_{2}}
$$

for all $g_{1} \preceq g_{2} \in G$. Equivalently, all diagrams of the following form commute:

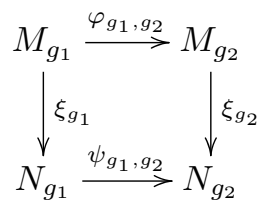

With such morphisms, the class of all GAPMs over $R$ becomes a category, which we call $R$-Persmod ${ }^{G}$.

Lemma 14 The maps $\alpha$ and $\beta$ from (4) and (5) extend to functors between $R$-Persmod ${ }^{G}$ and $R[G]-\mathbf{G r}$ - Mod which form an isomorphic pair of functors. In particular, the two categories are isomorphic.

The proof is similar to the proof of Lemma 4-see Appendix 2 for details. 
Finiteness conditions As in the case of linear sequences, we are interested in the subcategory of $R$-Persmod ${ }^{G}$ that is isomorphic to the category of finitely presented $R[G]$-modules. In order to describe the desired subcategory constructively, we introduce the notions of frames:

Definition 15 Let $\mathscr{M}_{G}=\left(\left(M_{g}\right)_{g \in G},\left(\varphi_{g_{1}, g_{2}}\right)_{g_{1} \leq g_{2} \in G}\right)$ be a $G A P M$. For $g \in G, h \in G$ is a frame of $g$ (w.r.t. $\mathscr{M}_{G}$ ) if $h \preceq g$ and for all $h \preceq \tilde{g} \preceq g$, the map $\varphi_{h, \tilde{g}}$ is an isomorphism. We say that $H \subseteq G$ is a framing set of $\mathscr{M}_{G}$ if every $g \in G$ has a frame in $H$. $\mathscr{M}_{G}$ is of finite type if there exists a finite framing set for $G$. It is called of finitely presented (generated) type if it is of finite type and each $M_{g}$ is finitely presented (generated) as an $R$-module.

From this definition, it follows that $e$ is an element in each framing set because of anti-symmetry. Moreover, trivially, each $\mathscr{M}_{G}$ has a framing set, namely $G$ itself. Another useful property is that if $g \in G$ and $h$ is a frame of $g$, then for every $\tilde{g}$ with $h \preceq \tilde{g} \preceq g, \varphi_{\tilde{g}, g}$ is an isomorphism as well, just because $\varphi_{\tilde{g}, g} \circ \varphi_{h, \tilde{g}}=\varphi_{h, g}$.

We discuss a related concept that is equivalent in some cases. Let $\mathscr{M}_{G}=\left(\left(M_{g}\right)_{g \in G},\left(\varphi_{g_{1}, g_{2}}\right)_{g_{1} \leq g_{2} \in G}\right)$ be a $G A P M$. We define $\left(g_{i}\right)_{i \in \mathbb{N}}$ to be a sequence in $G$ if $g_{i} \in G$ and $g_{i} \preceq g_{i+1}$ for all $i \in \mathbb{N}$. A sequence $\left(g_{i}\right)_{i \in \mathbb{N}}$ in $G$ induces a sequence $\left(M_{g_{i}}\right)_{i \in \mathbb{N}}$ in $\mathscr{M}_{G}$ with connecting morphisms $\varphi_{g_{i}, g_{i+1}}: M_{g_{i}} \rightarrow M_{g_{i+1}}$. We say that the latter sequence becomes stationary if there exists a $D \in \mathbb{N}$ such that $\varphi_{g_{i}, g_{i+1}}$ is an isomorphism for all $i \geq D$. Note that (stationary) sequences in a GAPM are (finite type) DAPMs.

Lemma 16 For a GAPM $\mathscr{M}_{G}=\left(\left(M_{g}\right)_{g \in G},\left(\varphi_{g_{1}, g_{2}}\right)_{g_{1} \leq g_{2} \in G}\right)$ of finite type, every sequence in $\mathscr{M}_{G}$ becomes stationary.

Proof Consider a sequence $\left(g_{i}\right)_{i \in \mathbb{N}}$ in $G$. Let $H=\left\{h_{1}, \ldots, h_{k}\right\}$ be a finite set that frames $\mathscr{M}_{G}$. Now, for every $g_{i}$, if $h_{j} \preceq g_{i}$, also $h_{j} \preceq g_{\ell}$ for $i \leq \ell$. Hence, setting $H_{i}:=\left\{h \in H \mid h \preceq g_{i}\right\}$, we get that $H_{0} \subseteq H_{1} \subseteq \ldots$, and since $H$ is finite, there is a $D \in \mathbb{N}$ such that $H_{D}=H_{D+1}=\cdots$.

Now, fix some $i \geq D$. Pick a frame $h \in H$ of $g_{i+1}$. By construction, $h \in H_{D}$ and hence, $h \preceq g_{i}$ as well. Hence, $h \preceq g_{i} \preceq g_{i+1}$, which implies that $\varphi_{g_{i}, g_{i+1}}$ is an isomorphism.

The converse of the statement is not true in general: Consider the monoid $\left(\mathbb{Q}_{\geq 0},+\right)$. The monoid is good, since the (unique) plcm of a finite subset is the maximal element in the set. We construct a GAPM $\mathscr{M}_{G}$ by setting $M_{0}:=R$ and $M_{q}:=0$ for all $q>0$ and let all maps be the zero maps. It is then obvious that every sequence becomes stationary. However, no finite subset can frame $\mathscr{M}_{G}$ because for every choice of finite subsets $\left\{0, q_{1}, \ldots, q_{D}\right\}$ with $q_{i}>0$ there are elements $q>0$ such that $q<q_{i}$ for all $i \in\{1, \ldots, D\}$.

In the previous example, we also observe that the corresponding $R\left[\mathbb{Q}_{\geq 0}\right]$-module is finitely generated. In some cases, however, both conditions are indeed equivalent: 
Lemma 17 If $G=\mathbb{N}^{k}$, a GAPM is of finite type if and only if every sequence becomes stationary.

Proof Fix $\mathscr{M}_{\mathbb{N}^{k}}=\left(\left(M_{g}\right)_{g \in \mathbb{N}^{k}},\left(\varphi_{g_{1}, g_{2}}\right)_{g_{1} \leq g_{2} \in \mathbb{N}^{k}}\right)$. Let $H \subset \mathbb{N}^{k}$ be a (not necessarily finite) set framing $\mathscr{M}_{\mathbb{N} k}$. We call $H$ reduced if there is no pair $h_{1} \neq h_{2} \in H$, such that $h_{1}$ is a frame of $h_{2}$. If $H$ is not reduced, and $h_{1} \leq h_{2}$ is a pair as above, it is not difficult to show that $H \backslash\left\{h_{2}\right\}$ is a framing set for $\mathscr{M}_{\mathbb{N}^{k}}$ as well. Moreover, for $G=\mathbb{N}^{k}$, every decreasing sequence of elements with respect to $\prec$ has a minimal element. That implies that there exists a reduced framing set for $\mathscr{M}_{\mathbb{N} k}$.

By the previous lemma, we know that if $\mathscr{M}_{\mathbb{N}^{k}}$ is of finite type, every sequence becomes stationary. For the converse, if $\mathscr{M}_{\mathbb{N}^{k}}$ is not of finite type, we choose a reduced framing set $H \subseteq \mathbb{N}^{k}$, which is necessarily infinite. We will now construct a non-stationary sequence iteratively, adding two elements to the sequence in each step. Set $g_{0}:=e \in \mathbb{N}^{k}$ and $H_{1}:=H \backslash\left\{g_{0}\right\}$; during the construction, $H_{i}$ will always be an infinite subset of $H$. For any such set $H_{i}$, by Dickson's Lemma (Rosales and Sánchez 1999, Theorem 5.1), there exists a finite subset $A_{i} \subset H_{i}$ such that for every $h \in H_{i}$, there exists an $a \in A_{i}$ with $a \preceq h$. Define $M_{i}(a):=\left\{h \in H_{i} \mid a \prec h\right\}$ for $a \in A_{i}$. Since $H_{i}$ is infinite, there exists at least one $g_{2 i} \in A_{i}$ such that $M_{i}\left(g_{2 i}\right)$ is infinite. Since $g_{2 i-2}$ is not a frame of $g_{2 i}$ (because they are both in $H$ ), there exists some $g_{2 i-1} \in \mathbb{N}^{k}$ such that $g_{2 i-2} \prec g_{2 i-1} \preceq g_{2 i}$ and $\varphi_{g_{2 i-2}, g_{2 i-1}}$ is not an isomorphism. We set $H_{i+1}:=M_{i}\left(g_{2 i}\right)$ and proceed with the next iteration. In this way, we obtain a sequence $\left(g_{i}\right)_{i \in \mathbb{N}}$ with $g_{i} \preceq g_{i+1}$ that does not become stationary.

We remark that the equivalence is not true in general as the aforementioned counterexample over $\left(\mathbb{Q}_{\geq 0},+\right)$ shows. In this case, a reduced framing set does not exist. Dually, the monoid $\left(\mathbb{N}^{\mathbb{N}},+\right)$ of all sequences of natural numbers yields an example for which the equivalence is not true either, but it is always possible to obtain reduced framing sets for GAPMs indexed over $\left(\mathbb{N}^{\mathbb{N}},+\right)$.

Let us now see that GAPMs of finitely presented type over $R$ are isomorphic to finitely presented graded $R[G]$-modules using the functors $\alpha$ and $\beta$. The next three lemmas generalize the corresponding statements in Sect. 3, with similar proof ideas.

Lemma 18 If a GAPM $\mathscr{M}_{G}=\left(\left(M_{g}\right)_{g \in G},\left(\varphi_{g_{1}, g_{2}}\right)_{g_{1} \leq g_{2} \in G}\right)$ is of finitely presented type, $\alpha\left(\mathscr{M}_{G}\right)$ is finitely presented.

Proof Fix $H=\left\{h_{1}, \ldots, h_{D}\right\}$ as finite set framing $\mathscr{M}_{G}$. Write $\mathfrak{G}_{i}$ for a finite generating set of $M_{h_{i}}$ and $n_{i}:=\left|\mathfrak{G}_{i}\right|$. Let $g \in G$ and $h_{i}$ be a frame of $g$. In particular, there exists some $f \in G$ such that $f \star h_{i}=g$, meaning that in $\alpha\left(\mathscr{M}_{G}\right)$, the map $f: M_{h_{i}} \rightarrow M_{g}$ is an isomorphism. Hence, the elements of $f \mathfrak{G}_{i}$ generate $M_{g}$. This shows that $\bigcup_{i=1}^{D} \mathfrak{G}_{i}$ generates $\alpha\left(\mathscr{M}_{G}\right)$.

For the second part, let $\mu_{i}: R^{n_{i}} \rightarrow M_{h_{i}}$ be the generating surjective map that corresponds to $\mathscr{G}_{i}$ where $i \in\{1, \ldots, D\}$. Writing $n=\sum_{i \bar{D}^{1}}^{D} n_{i}$, there is a map $\mu: R[G]^{n} \rightarrow \alpha(\mathscr{M})$ that corresponds to the generating set $\bigcup_{i=1}^{i} \mathfrak{G}_{i}$. If $\mathfrak{g}_{i}$ is a generator, we will use the notation $\mathfrak{e}_{i}$ to denote the corresponding generator of $R[G]^{n}$. 
We now define a finite set of elements of ker $\mu$. First of all, let $\boldsymbol{\bigotimes}_{i}$ be the generating set of ker $\mu_{i}$ for $0 \leq i \leq D$. Clearly, all elements of $\boldsymbol{Z}_{i}$ are also in ker $\mu$. Moreover, for $h_{i} \preceq h_{j}$ and any generator $\mathfrak{g}_{i}$ in $\mathfrak{G}_{i}$ with $\varphi_{h_{i}, h_{j}}\left(\mathfrak{g}_{i}\right) \neq 0$, we can write

$$
\varphi_{h_{i}, h_{j}}\left(\mathfrak{g}_{i}\right)=\sum_{v=1}^{n_{j}} \lambda_{\nu} \mathfrak{g}_{j}^{(v)}
$$

where $\lambda_{v} \in R$ and $\mathfrak{G}_{j}=\left\{\mathfrak{g}_{j}^{(1)}, \ldots, \mathfrak{g}_{j}^{\left(n_{j}\right)}\right\}$. In that case, the corresponding element

$$
f_{i, j} \mathbf{e}_{i}-\sum_{v=1}^{n_{j}} \lambda_{v} \mathbf{e}_{j}^{(v)}
$$

is in ker $\mu$ where $f_{i, j}$ is the unique element such that $f_{i, j} \star h_{i}=h_{j}$.

We let $\boldsymbol{Z}_{i, j}$ denote the finite set obtained by picking one element as above for each $\mathfrak{g}_{i}$ with $\varphi_{h_{i}, h_{j}}\left(\mathfrak{g}_{i}\right) \neq 0$. We claim that $\boldsymbol{Z}:=\bigcup_{i=0}^{D} \boldsymbol{Z}_{i} \cup \bigcup_{h_{i} \leq h_{j}} \boldsymbol{Z}_{i, j}$ generates ker $\mu$ :

Fix an element in $x \in \operatorname{ker} \mu$, which is of the form

$$
x=\sum_{\ell} \lambda_{\ell} e_{\ell}
$$

with $\lambda_{\ell} \in R[G]$ and $\mathbf{e}_{\ell}$ a generator of $R[G]^{n}$. We can assume that $x$ is homogeneous of some degree $g$. First we consider the case that $g=h_{j} \in H$ and all $\lambda_{\ell}$ are of degree $e$. Then, all $\mathrm{e}_{\ell}$ that appear in $x$ are of the same degree, and hence, their images under $\mu$ are generators of $M_{h_{j}}$. It follows that $x$ is generated by the set $\boldsymbol{Z}_{j}$.

Next, we consider the case that $g=h_{j} \in H$ and some $\lambda_{\ell}$ is of non-trivial degree. Because $x$ is homogeneous, $\lambda_{\ell}$ is then of the form $r_{\ell} f_{\ell, j}$ for some $r_{\ell} \in R$ and nontrivial $f_{\ell, j} \in G$. Since the degree of $\mathbf{e}_{\ell}$ is a $h_{\ell}$ with $f_{\ell, j} \star h_{\ell}=h_{j}$, there is an element $\mathfrak{z}_{\ell}$ in $\boldsymbol{Z}_{\ell, j}$ of the form $\mathfrak{z}_{\ell}=f_{\ell, j} \mathfrak{e}_{\ell}-\sum_{v=1}^{n_{\ell}} \tilde{\lambda}_{v} \mathfrak{e}_{j}^{(v)}$ with all $\mathfrak{e}_{j}^{(v)}$ of degree $h_{j}$ and each

$\tilde{\lambda}_{v} \in R$. Then, by turning from $x$ to $x-r_{\ell} z_{\ell}$, we turn the coefficient of $\mathfrak{e}_{\ell}$ into 0 and we only introduce summands with coefficients of degree $e$ in any non-trivial $g \in G$. Iterating this construction for each summand with coefficient of positive degree, we get an element $x^{\prime}=x-\sum_{w} r_{w} \mathfrak{z}_{w}$ with $r_{w} \in R$ and $\mathfrak{z}_{w}$ elements of $\mathbf{Z}$, and $x^{\prime}$ only having coefficients of degree $e$ in any non-trivial $g \in G$. By the first part, $x^{\prime}$ is generated by 3 , hence so is $x$.

Finally, let $g \notin H$. Then, there exists some $h_{i} \in H$ and some $f \in G$ such that $f \star h_{i}=g$ and multiplication with $f$ yields an isomorphism $M_{h_{i}} \rightarrow M_{g}$. Hence, we have that $x=f x^{\prime}$ with $x^{\prime}$ homogeneous of degree $h_{i}$. Since $0=\mu(x)=f \mu\left(x^{\prime}\right)$, it 
follows that $x^{\prime} \in \operatorname{ker} \mu$. By the above cases, $x^{\prime}$ is generated by $\mathbf{Z}$, and hence, so is $x$.

For the next two lemmas, we fix a finitely presented graded $R[G]$-module $\mathbf{M}:=\bigoplus_{g \in G} M_{g}$ with a map

$$
R[G]^{n} \stackrel{\mu}{\rightarrow} \mathbf{M}
$$

such that ker $\mu$ is finitely generated. Moreover, we let $\mathfrak{G S}:=\left\{\mathfrak{g}^{(1)}, \ldots, \mathfrak{g}^{(n)}\right\}$ denote generators of $\mathbf{M}$ and $\boldsymbol{Z}:=\left\{\boldsymbol{z}^{(1)}, \ldots, \boldsymbol{z}^{(m)}\right\}$ denote a generating set of ker $\mu$. We assume that each $\mathfrak{g}^{(i)}$ and each $z^{(j)}$ is homogeneous (with respect to the grading of the corresponding module), and we let $\operatorname{deg}\left(\mathfrak{g}^{(i)}\right), \operatorname{deg}\left(\mathfrak{z}^{(j)}\right)$ denote the degrees.

Lemma 19 Each $M_{g}$ is finitely presented as an R-module.

Proof We argue first that $M_{g}$ is finitely generated. Let $n_{g}$ denote the number of elements $\mathfrak{g}^{(j)}$ in $\mathfrak{G}$ such that $\operatorname{deg}\left(\mathfrak{g}^{(j)}\right) \preceq g$. Let $h_{j} \in G$ such that $h_{j} \star \operatorname{deg}\left(\mathfrak{g}^{(j)}\right)=g$. Define the map $\mu_{g}: R^{n_{g}} \rightarrow M_{g}$, by mapping the $j$ th generator $\mathbf{e}_{g}^{(j)}$ of $R^{n_{g}}$ to the element $h_{j} \mathfrak{g}^{(j)}$. Then the map $\mu_{g}$ is surjective, proving that $M_{i}$ is finitely generated.

We show that ker $\mu_{g}$ is finitely generated as well. Let $\mathfrak{e}^{(1)}, \ldots, \mathfrak{e}^{(n)}$ be the generators of $R[G]^{n}$ mapping to $\mathfrak{g}^{(1)}, \ldots, \mathfrak{g}^{(n)}$ under $\mu$. Let $m_{g}$ denote the number of elements in 3 such that $\operatorname{deg}\left(\mathfrak{z}^{(j)}\right) \preceq g$. Let $h_{j}^{\prime} \in G$ such that $h_{j}^{\prime} \star \operatorname{deg}\left(\mathfrak{z}^{(j)}\right)=g$. For every $\mathfrak{z}^{(j)}$

with $1 \leq j \leq m_{g}$, consider $h_{j}^{\prime} \mathfrak{z}^{(j)}$, which can be written as

$$
h_{j}^{\prime} \mathfrak{z}^{(j)}=\sum_{k=1}^{n_{g}} r_{k} h_{k} \mathfrak{e}^{(k)}
$$

with $r^{(k)} \in R$. Now, define

$$
\mathfrak{z}_{g}^{(j)}:=\sum_{k=1}^{n_{g}} r_{k} \mathfrak{e}_{g}^{(k)}
$$

and $\boldsymbol{Z}_{g}:=\left\{\boldsymbol{z}_{g}^{(j)} \mid 1 \leq i \leq m_{g}\right\}$. We claim that $\boldsymbol{Z}_{g}$ generates ker $\mu_{g}$. First of all, we get $\mu_{g}\left(z_{g}^{(j)}\right)=\mu\left(\mathfrak{z}^{(j)}\right)=0$. Now fix $x \in$ ker $\mu_{g}$ arbitrarily. Then, $x$ is a linear combination of elements in $\left\{\mathfrak{e}_{g}^{(1)}, \ldots, \mathfrak{e}_{g}^{\left(n_{g}\right)}\right\}$ with coefficients in $R$. Replacing $\mathfrak{e}_{g}^{(j)}$ with $h_{j} \mathfrak{e}^{(j)}$, we obtain $x^{\prime} \in R[G]^{n}$ homogeneous of degree $g$. By assumption, we can write $x^{\prime}$ as linear combination of elements in $\mathfrak{Z}$, that is,

$$
x^{\prime}=\sum_{k=1}^{m_{g}} r_{k}^{\prime} h_{k}^{\prime} \mathfrak{z}^{(k)}
$$


with $r_{k}^{\prime} \in R$. Then, it holds that

$$
x=\sum_{k=1}^{m_{g}} r_{k}^{\prime} \mathfrak{z}_{g}^{(k)}
$$

which follows by comparing coefficients: let $j \in\left\{1, \ldots, n_{g}\right\}$ and let $c_{j} \in R$ be the coefficient of $\mathbf{e}_{g}^{(j)}$ in $x$. Let $c_{j}^{\prime}$ be the coefficient of $\mathbf{e}_{g}^{(j)}$ in the sum $\sum_{k=1}^{m_{g}} r_{k}^{\prime} \mathbf{z}_{g}^{(k)}$, expanding

each $\mathfrak{z}_{g}^{(k)}$ by its linear combination as above. By construction, $c_{j}$ is the coefficient of $h_{k} \mathrm{e}^{(j)}$ in $x^{\prime}$, and $c_{j}^{\prime}$ is the coefficient of $h_{k} \mathrm{e}^{(j)}$ in the sum $\sum_{k=1}^{m_{i}} r_{k}^{\prime} h_{k}^{\prime} \mathfrak{z}^{(k)}$. Since this sum

equals $x^{\prime}$, it follows that $c_{j}=c_{j}^{\prime}$. Since $x$ was chosen arbitrarily from ker $\mu_{g}$, it fol-

lows that $\boldsymbol{Z}_{g}$ generates the kernel.

Lemma $20 \beta(\mathbf{M})$ is of finite type. In particular, it is of finitely presented type with Lemma 19.

Proof Define

$$
D:=\left\{\operatorname{deg}\left(\mathfrak{g}^{(j)}\right) \mid 1 \leq j \leq n\right\} \cup\left\{\operatorname{deg}\left(\mathfrak{z}^{(k)}\right) 1 \leq k \leq m\right\} .
$$

For every subset $D^{\prime}$ of $D$, let $p l c m\left(D^{\prime}\right)$ denote the set of partially least common multiples of $D^{\prime}$. Then, set

$$
H:=\bigcup_{D^{\prime} \subseteq D} \operatorname{plcm}\left(D^{\prime}\right)
$$

Note that $e \in H$ (using $D^{\prime}=\emptyset$ ) and $D \subset H$ (when $D^{\prime}$ ranges over the singleton sets). Also, $H$ is finite because $D$ is finite and $G$ is weak plcm.

We claim that $H$ frames $\beta(\mathbf{M})$. Let $g \in G$ be arbitrary. We have to find an element $h \in H$ such that $h \preceq g$ and for all $h \preceq \tilde{g} \preceq g$ with $\tilde{f} \star h=\tilde{g}$, multiplication with $\tilde{f}$ is an isomorphism $M_{h} \rightarrow M_{\tilde{g}}$.

If $g \in H$, that claim is trivial using $h:=g$ and $\tilde{f}:=e$. So, let us assume that $g \notin H$. Let $D^{\prime}(g):=\{\ell \in D \mid \ell \preceq g\}$. Then, $g$ is a common multiple of $D^{\prime}(g)$. However, it is not a partially least common multiple because in that case, it would belong to $H$. Hence, there exists a plcm $h$ of $D^{\prime}(g)$ such that $h \preceq g$. Note also that $D^{\prime}(g)=D^{\prime}(h)$ in this case. Let $h \preceq \tilde{g} \preceq g$ and $\tilde{f}$ be such that $\tilde{f} \star h=\tilde{g}$.

We first show that multiplication by $\tilde{f}$ is surjective. For that, fix some $x \in M_{\tilde{g}}$. Then $x$ is generated by a linear combination of the generators $\mathfrak{g}^{(j)}$, and among those, only those in $D^{\prime}(\tilde{g})=D^{\prime}(g)$ can have non-zero coefficients. Hence, $x$ takes the form

$$
x=\sum_{j \in\{1, \ldots, n\}, \operatorname{deg}\left(\mathfrak{g}^{(j)}\right) \in D^{\prime}(g)} f_{j} r_{j} \mathfrak{g}^{(j)}
$$


with $r_{j} \in R$ and $f_{j} \in G$ such that $f_{j} \star \operatorname{deg}\left(\mathfrak{g}^{(j)}\right)=\tilde{g}$. Let $f_{j}^{\prime}$ be such that $f_{j}^{\prime} \star \operatorname{deg}\left(\mathfrak{g}^{(j)}\right)=h$. Then, using $\tilde{f}$ from above,

$$
\tilde{f} \star f_{j}^{\prime} \star \operatorname{deg}\left(\mathfrak{g}^{(j)}\right)=\tilde{g}=f_{j} \star \operatorname{deg}\left(\mathfrak{g}^{(j)}\right),
$$

which implies that $\tilde{f} \star f_{j}^{\prime}=f_{j}$ because $G$ is right-cancellative. Hence we can write

$$
\begin{aligned}
x & =\sum_{j \in\{1, \ldots, n\}, \operatorname{deg}\left(\mathfrak{g}^{(j)}\right) \in D^{\prime}(g)}\left(\tilde{f} \star f_{j}^{\prime}\right) r_{j} \mathfrak{g}^{(j)} \\
& =\tilde{f} \sum_{j \in\{1, \ldots, n\}, \operatorname{deg}\left(\mathfrak{g}^{(j)}\right) \in D^{\prime}(g)} f_{j}^{\prime} r_{j} \mathfrak{g}^{(j)},
\end{aligned}
$$

which shows that $x$ has a preimage in $M_{h}$.

For the injectivity of multiplication with $\tilde{f}$, let $y \in M_{h}$ such that $\tilde{f} y=0$. Let $x \in R[G]^{n}$ such that $\mu(x)=y$. Then $\mu(\tilde{f} x)=\tilde{f} y=0$ and hence, $\tilde{f} x$ is generated by the $\mathfrak{z}^{(k)}$. Similar as in the first part of the proof, only such $\mathfrak{z}^{(k)}$ can appear whose degree lies in $D^{\prime}(g)$. Hence, writing

$$
\tilde{f} x=\sum_{k \in\{1, \ldots, m\}, \operatorname{deg}\left(\mathfrak{z}^{(k)}\right) \in D^{\prime}(g)} f_{k} r_{k} \mathfrak{z}^{(k)},
$$

we know that $f_{k} \star \operatorname{deg}\left(\mathfrak{z}^{(k)}\right)=\tilde{g}$. The same way as in the first part of the proof, we get that $f_{k}=\tilde{f} \star f_{k}^{\prime}$ with some $f_{k}^{\prime} \in G$. It follows that

$$
\tilde{f} x=\tilde{f} \sum_{k \in\{1, \ldots, m\}, \operatorname{deg}\left(\mathfrak{z}^{(k)}\right) \in D^{\prime}(g)} f_{k}^{\prime} r_{k} \mathfrak{z}^{(k)}
$$

Since $G$ is left-cancellative, $R[G]$ is left-cancellative with respect to multiplication by any monoid element. This property carries over to the free module $R[G]^{n}$ and it follows that

$$
x=\sum_{k \in\{1, \ldots, m\}, \operatorname{deg}\left(\mathfrak{z}^{(k)}\right) \in D^{\prime}(g)} f_{k}^{\prime} r_{k} \mathfrak{z}^{(k)} .
$$

Thus $x$ can be written as linear combination of the $z^{(k)}$, proving that $y=\mu(x)=0$.

The Generalized Representation Theorem The preceding lemmas imply the Generalized Representation Theorem: 
Theorem 21 Let $R$ be a ring with unity. The category of finitely presented graded $R[G]-m o d u l e s$ is isomorphic to the category of generalized algebraic persistence modules over $R$ of finitely presented type.

Proof The categories are subcategories of $R[G]-\mathbf{G r}-\operatorname{Mod}$ and $R$-Persmod ${ }^{G}$, respectively. Since $\alpha$ and $\beta$, restricted to these subcategories, map a GAPM of finitely presented type to a finitely presented graded $R[G]$-module (Lemma 18) and vice versa (Lemmas 19 and 20), these categories are isomorphic.

Clearly, this statement contains Theorem 9 using $G:=\mathbb{N}$.

As in Theorem 9, replacing "finitely presented" with "finitely generated" invalidates the claim. Passing to a Noetherian ring $R$, however, does not immediately revalidate it, because $R[G]$ is not Noetherian in general. Still, the following statement follows easily:

Corollary 22 If $R$ and $R[G]$ are Noetherian rings with unity, the category of finitely generated graded $R[G]$-modules is isomorphic to the category of discrete algebraic persistence modules of finitely generated type.

We remark that Hilbert's Basis Theorem can be applied iteratively (see Waerden 1993 , 15.1). Using $R\left[\mathbb{N}^{k}\right] \cong R\left[t_{1}, \ldots, t_{k}\right] \cong R\left[t_{1}, \ldots, t_{k-1}\right]\left[t_{k}\right]$, we obtain

Corollary 23 Let $R$ be a commutative Noetherian ring with unity, $k \in \mathbb{N}$. The category of finitely generated graded $R\left[\mathbb{N}^{k}\right]$-modules is isomorphic to the category of algebraic persistence modules over $\mathbb{N}^{k}$ of finitely generated type.

\section{Conclusion}

The Representation Theorem for persistence modules is one of the landmark results in the theory of persistent homology. In this paper, we formulated a more precise statement of this classical theorem over arbitrary rings with unity where we replaced finite generation with finite presentation. We provided a proof which only relies on elementary module theory. Furthermore, we generalized the Representation Theorem from naturally indexed modules to modules indexed over a more general class of monoids. The key difficulty was to find the right finiteness condition for persistence modules in this case to certify the equivalence with finitely presented modules graded over the monoid. Since the underlying ring does not have to be commutative, the Representation Theorem should rather be considered as a statement on general persistence, including the case of persistent homology.

Alternatively, in categorical language, persistence modules over $\mathbb{N}$ are functors $\mathbb{N} \rightarrow R$ - Mod where $\mathbb{N}$ is interpreted as the totally ordered set $(\mathbb{N}, \leq)$. For the Representation Theorem we used that $\mathbb{N}$ is a monoid and endowed the functor $\mathbb{N} \rightarrow R$ - Mod with an algebraic structure $R[t]$. We showed that the generalization of this situation is replacing $\mathbb{N}$ with a poset $(G, \preceq)$ that is induced by left factorization in 
a good monoid $(G, \star)$. Moreover, we defined the subcategory of finite-type-functors $G \rightarrow R$ - Mod precisely. The concept of frames enables us to locate the persistent features in the monoid concretely.

An obvious question is how the finiteness condition changes when the requirements on the monoid are further relaxed. The anti-symmetry of the monoid is not a severe assumption because elements $g_{1}, g_{2}$ with $g_{1} \preceq g_{2}$ and $g_{2} \preceq g_{1}$ induce isomorphic connecting maps in the corresponding GAPM and therefore can be treated as one component of the monoid. Also, we can relax the right-cancellative condition such that between any two elements $g_{1}, g_{2} \in G$, there are at most finitely many $h$ such that $h \star g_{1}=g_{2}$. We restricted to good monoids for clarity of exposition. If applications occur that require filtrations with some complicated ordering structure, we suggest to consider a good monoid as underlying index set.

A next step is to find classification results, parametrizations and discrete invariants for finitely presented monoid-graded modules. It might be useful to consider a subclass of good monoids, e.g., finitely generated good monoids or monoids with a manageable factorization theory. The theory of persistence modules is well-studied in the case of $\mathbb{N}^{k}$ and might be transferred to more general monoids, as much as the situation of discrete invariants. Since there is no natural notion of barcodes in the case of $\mathbb{N}^{k}$, there is also no hope of finding barcodes for more general monoids. But it might be possible to generalize a discrete invariant from $\mathbb{N}^{k}$, for instance the discrete rank invariant, and generalize algorithms to $G$-indexed persistence.

Acknowledgements Open access funding provided by Graz University of Technology. The authors are supported by the Austrian Science Fund (FWF) Grant number P 29984-N35.

Open Access This article is distributed under the terms of the Creative Commons Attribution 4.0 International License (http://creativecommons.org/licenses/by/4.0/), which permits unrestricted use, distribution, and reproduction in any medium, provided you give appropriate credit to the original author(s) and the source, provide a link to the Creative Commons license, and indicate if changes were made.

\section{Appendix 1: Details from Sect. 3}

Proof of functoriality of $\alpha$ Recall that for $\xi_{*}=\left(\xi_{i}\right)_{i \in \mathbb{N}}$, a DAPM morphism between $\mathscr{M}$ and $\mathcal{N}$, we define $\alpha\left(\xi_{*}\right)$ as the map assigning to $\left(m_{i}\right)_{i \in \mathbb{N}} \in \oplus M_{i}$ the value $\left(\xi_{i}\left(m_{i}\right)\right)_{i \in \mathbb{N}}$. Let us define $f:=\alpha\left(\xi_{*}\right)$ for shorter notation. Indeed, $f$ is a graded module morphism: it is a group homomorphism, as each $\xi_{i}$ is, it clearly satisfies $f\left(M_{i}\right) \subset N_{i}$, and it holds that for $m=\left(m_{0}, m_{1}, \ldots\right)$ we get

$$
\begin{aligned}
f(t m) & =f\left(0, t m_{0}, t m_{1}, \ldots\right)=\left(0, \xi_{1}\left(t m_{0}\right), \xi_{2}\left(t m_{1}\right), \ldots\right) \\
& =\left(0, t \xi_{0}\left(m_{0}\right), t \xi_{1}\left(m_{1}\right), \ldots\right)=t\left(\xi_{i}\left(m_{i}\right)\right)_{i \in \mathbb{N}}=t f(m),
\end{aligned}
$$


where the third equality comes from the property of DAPM morphisms.

For the functorial properties, it is clear that $\alpha$ maps the identity $D A P M$ morphism to the identity of the corresponding graded modules. If $\xi_{*}, \xi_{*}^{\prime}$ are DAPM morphisms and $m$ as before, we calculate

$$
\begin{aligned}
\left(\alpha\left(\xi_{*}^{\prime} \circ \xi_{*}\right)\right)(m) & =\left(\xi_{i}^{\prime}\left(\xi_{i}\left(m_{i}\right)\right)\right)_{i \in \mathbb{N}} \\
& =\alpha\left(\xi_{*}^{\prime}\right)\left(\xi_{i}\left(m_{i}\right)\right)_{i \in \mathbb{N}} \\
& =\left(\alpha\left(\xi_{*}^{\prime}\right) \circ \alpha\left(\xi_{*}\right)\right)(m) .
\end{aligned}
$$

Proof of functoriality of $\beta$ Fix two graded $R[t]$-modules $\oplus M_{i}$ and $\oplus N_{i}$ and let $\mathscr{M}:=\beta\left(\oplus M_{i}\right)$ and $\mathcal{N}:=\beta\left(\oplus N_{i}\right)$. A graded morphism $\eta: \oplus M_{i} \rightarrow \oplus N_{i}$ implies a sequence of maps $\left(\xi_{i}\right)_{i \in \mathbb{N}}:=\beta(\eta)$ where $\xi_{i}$ is just the restriction of $\eta$ to $M_{i}$. It follows that $\beta$ gives group homomorphisms $\xi_{i}: M_{i} \rightarrow N_{i}$ which are also $R$-module morphisms because for $r \in R, m_{i} \in M_{i}$ we get $\xi_{i}\left(r m_{i}\right)=\eta\left(r m_{i}\right)=r \eta\left(m_{i}\right)=r \xi_{i}\left(m_{i}\right)$. Since the connecting maps in $\mathscr{M}$ and $\mathscr{N}$ are induced by multiplication with $t$, we have that for $m_{i} \in M_{i}$,

$$
\xi_{i+1}\left(t m_{i}\right)=\eta\left(t m_{i}\right)=t \eta\left(m_{i}\right)=t \xi_{i}\left(m_{i}\right)
$$

proving that $\beta(\eta)$ is indeed a DAPM morphism.

For functoriality, it is again clear that $\beta$ maps the identity to the identity morphism. For two graded $R[t]$-module morphisms $\eta, \eta^{\prime}$ and any sequence $\left(m_{i}\right)_{i \in \mathbb{N}}$ with $m_{i} \in M_{i}$ for all $i \in \mathbb{N}$, we get

$$
\begin{aligned}
\left(\beta\left(\eta^{\prime} \circ \eta\right)\right)\left(m_{i}\right)_{i \in \mathbb{N}} & =\left(\eta^{\prime}\left(\eta\left(m_{i}\right)\right)_{i \in \mathbb{N}}\right. \\
& =\beta\left(\eta^{\prime}\right)\left(\eta\left(m_{i}\right)\right)_{i \in \mathbb{N}} \\
& =\left(\beta\left(\eta^{\prime}\right) \circ \beta(\eta)\right)\left(m_{i}\right)_{i \in \mathbb{N}}
\end{aligned}
$$

\section{Appendix 2: Details from Sect. 5}

Proof of Lemma 14 For a GAPM morphism $\left(\xi_{g}: M_{g} \rightarrow N_{g}\right)_{g \in G}$ between two GAPMs $\mathscr{M}_{G}$ and $\mathscr{N}_{G}$, we define

$$
\alpha\left(\xi_{G}\right): \bigoplus_{g \in G} M_{g} \rightarrow \bigoplus_{g \in G} N_{g},\left(m_{g}\right)_{g \in G} \mapsto\left(\xi_{g}\left(m_{g}\right)\right)_{g \in G} .
$$


Recall that for $\xi_{G}=\left(\xi_{g}\right)_{g \in G}$, a $G A P M$ morphism between $\mathscr{M}_{G}$ and $\mathcal{N}_{G}$, we define $\alpha\left(\xi_{G}\right)$ as the map assigning to $\left(m_{g}\right) \in \oplus M_{g}$ the value $\left(\xi_{g}\left(m_{g}\right)\right)$. Let us define $f:=\alpha\left(\xi_{G}\right)$ for shorter notation. Again, $f$ is a graded module morphism: it is a group homomorphism, as each $\xi_{g}$ is, it clearly satisfies $f\left(M_{g}\right) \subset N_{g}$, and it holds that for $m=\left(m_{g}\right)_{g \in G}$ and $h \in G$ we get

$$
\begin{aligned}
& f(h m)=f\left(\left(\begin{array}{ll}
h m_{\tilde{g}} ; & \exists \widetilde{g} \in G: h \star \widetilde{g}=g \\
0 ; & \text { otherwise }
\end{array}\right)_{g \in G}\right) \\
& \left.=\left(\begin{array}{ll}
\xi_{h \star \widetilde{g}}\left(h m_{\tilde{g}}\right) ; & \exists \widetilde{g} \in G: h \star \widetilde{g}=g \\
0 ; & \text { otherwise }
\end{array}\right)_{g \in G}\right) \\
& \left.=\left(\begin{array}{ll}
h \xi_{\widetilde{g}}\left(m_{\widetilde{g}}\right) ; & \exists \widetilde{g} \in G: h \star \widetilde{g}=g \\
0 ; & \text { otherwise }
\end{array}\right)_{g \in G}\right) \\
& \left.=\left(\begin{array}{ll}
h \xi_{\widetilde{g}}\left(m_{\tilde{g}}\right) ; & \exists \widetilde{g} \in G: h \star \widetilde{g}=g \\
0 ; & \text { otherwise }
\end{array}\right)_{g \in G}\right) \\
& =h\left(\xi_{g}\left(m_{g}\right)\right)_{g \in G} \\
& =h f(m) \text {, }
\end{aligned}
$$

where the third equality comes from the property of GAPM morphisms.

For the functorial properties, it is clear that $\alpha$ maps the identity GAPM morphism to the identity of the corresponding graded module. If $\xi_{G}, \xi_{G}^{\prime}$ are GAPM morphisms and $m$ as before, we calculate

$$
\begin{aligned}
\left(\alpha\left(\xi_{*}^{\prime} \circ \xi_{*}\right)\right)(m) & =\left(\xi_{g}^{\prime}\left(\xi_{g}\left(m_{g}\right)\right)\right)_{g \in G} \\
& =\alpha\left(\xi_{*}^{\prime}\right)\left(\xi_{g}\left(m_{g}\right)\right)_{g \in G} \\
& =\left(\alpha\left(\xi_{*}^{\prime}\right) \circ \alpha\left(\xi_{*}\right)\right)(m) .
\end{aligned}
$$

Vice versa, a morphism

$$
\eta: \bigoplus_{g \in G} M_{g} \rightarrow \bigoplus_{g \in G} N_{g}
$$

in $R[G]-\mathbf{G r}-$ Mod induces a homomorphism $\eta_{g}: M_{g} \rightarrow N_{g}$ for each $g \in G$, and these induced maps are compatible with multiplication of each $h \in G$. Hence, the diagram 


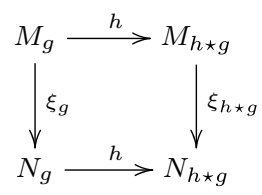

commutes and so, setting $\beta(\eta):=\left(\eta_{g}\right)_{g \in G}$ yields a $G A P M$ morphism between $\beta\left(\bigoplus_{g \in G} M_{g}\right)$ and $\beta\left(\bigoplus_{g \in G} N_{g}\right)$.

For functoriality, it is clear that $\beta$ maps the identity between graded modules to the identity GAPM morphism. For two graded $R[G]$-module morphisms $\eta, \eta^{\prime}$ and any $\left(m_{g}\right)_{g \in G}$ with $m_{g} \in M_{g}$ for all $g \in G$, we get

$$
\begin{aligned}
\left(\beta\left(\eta^{\prime} \circ \eta\right)\right)\left(m_{g}\right)_{g \in G} & =\left(\eta^{\prime}\left(\eta\left(m_{g}\right)\right)_{g \in G}\right. \\
& =\beta\left(\eta^{\prime}\right)\left(\eta\left(m_{g}\right)\right)_{g \in G} \\
& =\left(\beta\left(\eta^{\prime}\right) \circ \beta(\eta)\right)\left(m_{g}\right)_{g \in G} .
\end{aligned}
$$

Finally, the construction immediately implies that $\alpha \circ \beta$ equals the identity functor on $R[G]-\mathbf{G r}-\operatorname{Mod}$ and $\beta$ o $\alpha$ equals the identity functor on $R$-Persmod ${ }^{G}$, hence $(\alpha, \beta)$ is an isomorphic pair of functors.

\section{Appendix 3: Proving the ZC-representation theorem using Artin- Rees theory}

We showed how to prove the Representation Theorem over general rings with unity and good monoids as index set. Artin-Rees theory is defined over commutative Noetherian rings with unity and filtrations of subsets of modules. Therefore it does not yield a proof for more general rings or, at least not immediately, for more general indexing monoids than $\mathbb{N}$. Since Artin-Rees theory is quoted in Zomorodian (2009) and Zomorodian and Carlsson (2005) to provide a proof for Theorem 9 (over commutative Noetherian rings with unity), we take a closer look at the connections between Artin-Rees theory and such a proof. To do this, we consider the following notion of filtrations of modules:

Definition 24 Let $A$ be a commutative Noetherian ring with unity. Let $I \subseteq A$ be an ideal, $M$ an $A$-module. An $I$-filtration of $M$ is a collection $\left(M_{n}\right)_{n \in \mathbb{N}}$ such that $M=M_{0} \supseteq M_{1} \supseteq M_{2} \supseteq \cdots$ and $I M_{n} \subseteq M_{n+1}$ for all $n \in \mathbb{N}$. An $I$-filtration is called $I$-stable if there is an $n_{0} \in \mathbb{N}$ such that $I M_{n}=M_{n+1}$ for all $n \geq n_{0}$.

Now, consider an ideal $I \subseteq A$ and an $I$-filtration $\left(M_{n}\right)_{n \in \mathbb{N}}$ of a finitely generated $A$-module $M$. Let $\bar{A}:=\bigoplus_{i \in \mathbb{N}} I^{i}, \bar{M}:=\bigoplus_{i \in \mathbb{N}} M_{i}$. We obtain a criterion for $\left(M_{n}\right)_{n \in \mathbb{N}}$ to be I-stable: 
Lemma 25 (Criterion for stability) Let $A$ be a commutative Noetherian ring with unity, $I \subseteq A$ an ideal. Let $M$ be a finitely generated A-module, $\left(M_{n}\right)_{n \in \mathbb{N}}$ an I-filtration of $M$. Then the following are equivalent:

\section{1. $\bar{M}$ is a finitely generated $\bar{A}$-module.}

2. $\left(M_{n}\right)_{n \in \mathbb{N}}$ is I-stable.

This criterion helps to prove the famous Artin-Rees Lemma. It states that given a stable filtration of a finitely generated module $M$ and a submodule $N$ of $M$, then intersecting each member of the filtration with $N$ again yields a stable filtration. The Artin-Rees Lemma can for instance be used to prove Krull's Intersection Theorem (Eisenbud 1995) and to study modules over local rings (Greuel and Pfister 2007). For a proof of the above criterion, the Artin-Rees Lemma and the connection between the two lemmas, we refer to Greuel and Pfister (2007).

Let us see how this helps to prove the ZC-Representation Theorem for a commutative Noetherian ring $R$ with unity. Setting $A=R[t]$ and $I$ as the ideal generated by $t$, we obtain $\bar{A}=R[t]$. By Lemma $1, R[t]$ is Noetherian and finite presentation and finite generation coincide not only for $R$-modules, but also for $R[t]$-modules.

Let $\mathscr{M}$ be a $D A P M$ over $R$ of finitely generated type. Consider the filtration $\left(\widetilde{M}_{n}\right)_{n \in \mathbb{N}}$ defined by $\widetilde{M}_{0}=\bigoplus_{i \in \mathbb{N}} M_{i}$ and $\widetilde{M}_{n}=\left(t^{n}\right) \widetilde{M}_{0}$ for $n>0$. By finite type assumption, there exists an $n_{o} \in \mathbb{N}$ such that $(t) \widetilde{M}_{n}=\widetilde{M}_{n+1}$ for all $n \geq n_{0}$. To use the above criterion, we have to ensure that $\bigoplus_{i \in \mathbb{N}} M_{i}$ is a finitely generated $R[t]$-module. We do not see how this could follow from Artin-Rees theory since finite generation is an assumption in most of the statements. A proof for the finite generation of $\bigoplus_{i \in \mathbb{N}} M_{i}$ is the first part of our proof of Lemma 6.

Conversely, using the above criterion, finite generation of the $R[t]$-module $\bigoplus_{i \in \mathbb{N}} M_{i}$ directly implies $(t)$-stability of the filtration $\left(\widetilde{M}_{n}\right)_{n \in \mathbb{N}}$. Hence the corresponding $D A P M \beta\left(\bigoplus_{i \in \mathbb{N}} M_{i}\right)$ is of finite type. It is left to prove that each $M_{i}$ is finitely generated as an $R$-module. This can easily be seen by the first five lines of our proof of Lemma 7.

\section{References}

Adámek, J., Herrlich, H., Strecker, G.E.: Abstract and Concrete Categories-The Joy of Cats. Dover Publications, New York (2009)

Baeth, N.R., Smertnig, D.: Factorization theory: from commutative to noncommutative settings. J. Algebra 441, 475-551 (2015)

Bubenik, P., de Silva, V., Scott, J.: Metrics for generalized persistence modules. Found. Comput. Math. 15(6), 1501-1531 (2015)

Bubenik, P., Scott, J.: Categorification of persistent homology. Discret. Comput. Geom. 51(3), 600-627 (2014)

Carlsson, G.: Topology and data. Bull. AMS 46, 255-308 (2009)

Carlsson, G., Zomorodian, A.: The theory of multidimensional persistence. Discret. Comput. Geom. 42(1), 71-93 (2009)

Chazal, F., de Silva, V., Glisse, M., Oudot, S.: The Structure and Stability of Persistence Modules. Springer, Berlin (2016) 
Clifford, A.H., Preston, G.B.: The Algebraic Theory of Semigroups. Mathematical Surveys and Monographs. American Mathematical Society, Providence (1961)

Corbet, R.: Verallgemeinerte Persistenzmoduln. Master's thesis, Technische Universität Berlin (2016)

Crawley-Boevey, W.: Decomposition of pointwise finite-dimensional persistence modules. J. Algebra Appl. 14(05), 1550066 (2015). https://doi.org/10.1142/S0219498815500668

Dehornoy, P.: Braids and Self-Distributivity. Springer, Berlin (2000)

Dehornoy, P., Digne, F., Godelle, E., Krammer, D., Michel, J.: Foundations of Garside Theory, vol. 22. European Mathematical Society, London (2015)

Dehornoy, P., Paris, L.: Gaussian groups and Garside groups, two generalisations of Artin groups. Proc. Lond. Math. Soc. 79(3), 569-604 (1999)

Droste, M., Kuske, D.: Recognizable languages in divisibility monoids. Math. Struct. Comput. Sci. 11(6), 743-770 (2001)

Edelsbrunner, H., Harer, J.: Computational Topology. An Introduction. American Mathematical Society, Providence (2010)

Edelsbrunner, H., Letscher, D., Zomorodian, A.: Topological persistence and simplification. Discret. Comput. Geom. 28(4), 511-533 (2002). https://doi.org/10.1007/s00454-002-2885-2

Edelsbrunner, H., Morozov, D.: Persistent homology: theory and practice. In: Proceedings of the European Congress of Mathematics. European Mathematical Society, pp. 31-50 (2012)

Eisenbud, D.: Commutative Algebra: With a View Toward Algebraic Geometry. Graduate Texts in Mathematics. Springer, Berlin (1995)

Geroldinger, A., Halter-Koch, F.: Non-Unique Factorizations: Algebraic, Combinatorial and Analytic Theory. Pure and Applied Mathematics. Chapman and Hall/CRC, London (2006)

Ghrist, R.: Barcodes: the persistent topology of data. Bull. AMS 45, 61-75 (2008)

Greuel, G., Pfister, G.: A Singular Introduction to Commutative Algebra. Incorporated, 2nd edn. Springer, Berlin (2007)

Grillet, P.A.: Semigroups: An Introduction to the Structure Theory. CRC Press, Boca Raton (2017)

Kerber, M.: Persistent homology: state of the art and challenges. Internationale Mathematische Nachrichten 231, 15-33 (2016)

Kuske, D.: Divisibility monoids: presentation, word problem, and rational languages. In: Freivalds, R. (ed.) Fundamentals of Computation Theory, pp. 227-239. Springer, Berlin (2001)

Lam, T.Y.: Lectures on Modules and Rings. Graduate Texts in Mathematics. Springer, New York (1999)

Mac Lane, S.: Categories for the Working Mathematician. Springer, Berlin (1971)

Oudot, S.: Persistence Theory: From Quiver Representation to Data Analysis. Mathematical Surveys and Monographs, vol. 209. American Mathematical Society, Providence (2015)

Picantin, M.: Garside monoids vs divisibility monoids. Math. Struct. Comput. Sci. 15(2), 231-242 (2005)

Rosales, J.C., García-Sánchez, P.A.: Finitely Generated Commutative Monoids. Nova Science Publishers, New York (1999)

Vejdemo-Johansson, M.: Interleaved equivalence of categories of persistence modules (2012). arXiv: 1210.7913

Vejdemo-Johansson, M.: Sketches of a platypus: persistent homology and its algebraic foundations. In: Algebraic topology: applications and new directions. Contemporary Mathematics, vol. 620, pp. 295-320. American Mathematical Society (2014)

van der Waerden, B.L.: Algebra II. Springer, Berlin (1993)

Wehrung, F.: Tensor products of structures with interpolation. Pac. J. Math. 176(1), 267-285 (1996)

Zomorodian, A.: Topology for Computing. Cambridge University Press, Cambridge (2009)

Zomorodian, A., Carlsson, G.: Computing persistent homology. Discret. Comput. Geom. 33(2), 249-274 (2005) 